\title{
SPREADSHEET PROGRAM FOR DETERMINATION OF RESERVOIR CHARACTERISTICS AND INFLOW PERFORMANCE RELATIONSHIP OF WELLS BY ANALYSING PRESSURE TRANSIENT TESTS
}

\author{
B. C. Jain, Abdurahman Kutty and S. K. Patra \\ Institute of Reservoir Studies, Oil \& Natural Gas Corporation Ltd., Chandkheda Campus, \\ Ahmedabad - 380 005, India
}

(Received: 1 November 1995; Accepted: 31 January 1996)

\begin{abstract}
Importance of pressure transient analysis during preparation of development schemes and performance analysis of oil/gas reservoirs is accredited in oil and gas industry due to its usage in estimation of petrophysical parameters of the reservoir and analysis of in flow performance relationship of oil wells, open flow performance of gas wells etc.

In general, data analysis may be stated as a two-fold process: (i) data management, se lection and calculations and (ii) plotting of the required data. Most of the available PC based software packages for pressure transient analysis perform complex calculations but are defi cient in their data management / graphic output capabilities which usually is rigid and limit ed. Based on the popular spreadsheet software package Quattro Pro ${ }^{\mathrm{TM}}$, a powerful and time saving spreadsheet program for pressure transient analysis is presented. The spreadsheet prepared is simple to use and can be customised to user's needs by modifying the given for mat with a provision of further expansion.
\end{abstract}

Key Words : Spreadsheet program, Oil and Gas Reservoir, Pressure transient test

\section{INTRODUCTION}

One of the important goals of reservoir engineer ing is the attainment of maximum efficiency in the exploitation of oil/gas reservoirs leading to maximum recovery (Nolen-Hoeksema, 1990). This requires that the limits and heterogeneity of reservoirs is properly understood to work out an optimum de velopment plan and to implement enhanced recovery methods. Estimating the unknown properties and a detailed reservoir description is critical in a mathe matical reservoir simulation to give a best fit to the measured performance and for future performance predictions (Lee et al., 1986; Kossack, 1989).

It is well known since long that reservoir rocks are heterogeneous at megascopic (kilometer-scale), macroscopic (meter-scale) and microscopic levels. At least for the time being, however, we know very little about these variations and are unable to modify this state of non-homogeneity. Pressure transient tests constitute one of the important tools used for predicting the heterogeneity of reservoirs. Analysis of such test data provides useful information about reservoir extent, formation permeability, fluid pres sure, skin (wellbore conditions), flow efficiency etc.

Pressure transient tests requires that the flow rate of a well be changed and the associated changes in bottomhole pressure measured as a function of time. The resulting data provides valuable information for a comprehensive reservoir analysis. These tests are advantageous over many other well testing techniques, most important being that it can be run at any time during the life of a well. A single test can give the data required to infer the desired reservoir information. Further, the technique is applicable in oil, gas or water wells, fluid/air injection wells or hot water and steam wells.

Unfortunately, pressure transient tests are influ enced by several factors that can invalidate part, or all of the test data. A care must, therefore, be taken not only about the stress for accuracy but also for what if situations.

The spreadsheet developed here makes use of the transient tests data to make various calculations and simultaneously generates plots pertaining to pressure buildup, pressure drawdown, two - rate test, influx study, inflow performance tests, isochronal test, pressure fall - off test in water injection and in - situ combustion wells, etc.

\section{THE DATABASE}

An electronic spreadsheet is a table made up of columns and rows, where each intersection constitute a cell, making the computer into a giant ledger sheet. Each cell stores a data value or a formula. Whereas a ledger sheet used in accounting is limited in their capacity the electronic spreadsheet is have a maximum size of 234 columns and 8192 rows.

The cells of spreadsheet prepared here can be di vided basically into 2 parts. One set contains the basic input data required and the other set is used for cal culations. This way each cell contains a data pertain ing to pressure, time, formation parameters, oil/gas constants, or a formula. Various graphs are pre pared and stored in the spreadsheet with the built - in graphics facility using the above sets of data. 


\section{DATA MANAGEMENT}

Data management in the spreadsheet not only utilizes the most important features of electronic spreadsheet (ability of automatic incorporation of all the required changes in data and the graphs prepared in the spreadsheet, if even one value changes), but al so provides wide applicability along with other facili ties.

For preparing the spreadsheet, after invoking the worksheet in Quattro Pro ${ }^{\mathrm{TM}}$, data on deflection, divi sion, time, pressure, various constants etc. along with different formulae are entered at appropriate places in the required cells for performing calcula tions pertaining to the methods used in transient test ing. These formulae can be copied up to desired number of rows depending upon the requirement of input data. Design of formulae is limited only by user imagination; one can invoke any simple or multiple logical functions and apply it to any of the fields.

\section{GRAPHIC PRESENTATION}

Built - in graphics routines allow up to six ranges of $\mathrm{Y}$ data to be plotted against the $\mathrm{X}$ range on a cartesian / semi-log / log-log diagram. Various graphs with different graphics settings can be made, stored and recalled. Portions of the graphs can be analysed microscopically using range and scale facil ities. Titles and legends can be typed directly on a spread sheet cell. High quality graphics can be produced with the spreadsheet, including full color ones. The graphics also can be exported to drafting soft ware in order to produce publication - ready laser or dot matrix prints.

\section{PRESENT WORK}

Application area of the developed spreadsheet covers up related calculations and various plots pertaining to pressure build - up, pressure drawdown, two - rate test, influx study, inflow performance test, isochronal test, pressure fall - off test in in - situ com bustion processes and pressure fall - off test in water injection wells. These plots are Zero time pressure correction plot, Diagnostic plot, MDH plot, Horner plot, Muskat plot, Semi - steady state analysis plot, Two - rate test plot, Plot for static reservoir pressure and productivity index from influx study, Plot for se lection of optimum bean size for oil wells, Plot for open flow potential for gas wells (pressure square approach), Plot for isochronal test, Plot for calcula tion of burnt volume by pressure fall - off test in in situ combustion wells and plot for pressure fall - off test in water injection wells. This spreadsheet also calculates results of various methods if basic data from the graphs is entered at appropriate places after drawing the semi - steady state /required lines for de termining reservoir parameters from the graphs used for calculation of results.

\section{CONCLUSION}

The program developed here was used for pro- cessing transient pressure data. Once the spreadsheet is ready, it does calculations and make graphs as quickly as the new data along with the required constant is entered at the appropriate places. Calculation accuracy with the spreadsheet is far better than mea surement precision.

The program is simple to use and can be cus tomised to user needs by modifying the given format, with a capability of further expansion to accommo date wide range of calculations and plots.

\section{ACKNOWLEDGEMENT}

Authors are thankful to ONGC for rendering fa cilities to complete the work and for permission to present the paper in IAMG - 1995 Conference at Osa ka, Japan.

\section{REFERENCES}

Kossack, A. C. (1989) Prediction of layer lengths from layer heights for reservoir simulation: A statistical analysis of outcrop data. Jour. Petrol. Tech., August 1989, pp. $867-871$.

Lee, T., Kravaris, C., and Seinfeld, J. H. (1986) His tory matching by spline approximation and regu larization in single - phase areal reservoirs. $S P E$ Reservoir Engineering, September 1986, pp. 521 534.

Nolen-Hoeksema, R. C. (1990) The future role of geophysics in reservoir engineering. Geophysics: The leading edge of Exploration, December 1990, pp. 89 - 97.

\section{APPENDIX}

\section{SPREADSHEET LISTING}

A1: [W23] 'SPREADSHEET PROGRAM FOR DE TERMINATION OF RESERVOIR CHARACTERISTICS

A2: [W23] ' AND INFLOW PERFORMANCE RE LATIONSHIP OF WELLS BY ANALYSING

A3: [W23]' TESTS

PRESSURE TRANSIENT

J6: (F2) 'ZERO TIME

K6: [W12]' OIL

D7: [W12] `Clk. Const.

E7: [W10] "Mano. Const.

G7: [W11] 'Measuring Depth

I7: $[\mathrm{W} 12]{ }^{\wedge}$ Flow Time

J7: (F2) 'PR.ERROR

K7: [W12] ^ Rate

L7: `Q2/Q1

N7: [W11] ^ P1 - cap

O7: [W11] ^ P2 - cap

P7: [W11] ^ P3 - cap

D8: [W12] 0.0144

E8: [W10] 81.13

G8: [W11] 900

H8: [W12] 'm

I8: (F2) [W12] 165 
J8: (F2) 0

K8: (F2) [W12] 15.04

L8: (F2) +K9/K8

N8: (F2) [W11] 1290

O8: (F2) [W11] 1390

P8: (F2) [W11] 1450

K9: (F2) [W12] 0

D12: (F2) [W12] ^ D

E12: (F2) [W10] ${ }^{\wedge} \mathrm{E}$

F12: (F2) [W12] ${ }^{\wedge} \mathrm{F}$

G12: (F2) [W11] ^ $\mathrm{G}$

H12: [W12] ${ }^{\wedge} \mathrm{H}$

I12: [W12] ^ I

J12: (F2) ` J

K12: [W12] ` $\mathrm{K}$

L12: ` L

M12: [W11] ` $\mathrm{M}$

N12: [W11] ^ $\mathrm{N}$

012: [W11] ^ $\mathrm{O}$

P12: [W11]' $\mathrm{P}$

Q12: [W10]' Q

R12:' R

D13: (F2) [W12] ^ DEL. TIME

E13: (F2) [W10] ^ PRESSURE

F13: (F2) [W12] ` DEL. TIME

G13: (F2) [W11] ^ PRESSURE

H13: [W12] ^ PRESSURE

I13: [W12] ^ DEL - P

J13: (F2) ^ COR.DEL - P

K13: [W12] ^ HORNER

L13: ` TWO RATE

M13: [W11] 'LIN. ANA.

N13: [W11] ^ MUSKAT PR.

013: [W11] `MUSKAT PR.

P13: [W11] `MUSKAT PR.

Q13: (F2) [W10] 'DEL TIME

R13: 'WATER INJ.

D14: (F2) [W12] `div.

E14: (F2) [W10] $\wedge \mathrm{mm}$

F14: (F2) [W12] ^ $\mathrm{hr}$

G14: (F2) [W11] ^ $\mathrm{kg} / \mathrm{cm} 2$

H14: [W12] ^ $\mathrm{psi}$

I14: [W12] psi

J14: (F2) ^ psi

K14: [W12] ^ time

L14: ` time

M14: [W11] ^time

N14: [W11] ^psi

014: [W11] ^ psi

P14: [W11] ^ psi

Q14: (F2) [W10] 'MIN*1 00

R14:' time

A15: [W23] 'TYPE OF STUDIES

D16: (F4) [W12] 0

E16: (F4) [W10] 1.029

F16: (F4) $[\mathrm{W} 12]+\$ D \$ 8^{*} \mathrm{D} 16$

G16: (F2) [W11] + $\$ \mathrm{E} \$ 8^{*} \mathrm{E} 16$

H16: (F2) $[\mathrm{W} 12]+\$ \mathrm{E} \$ 8^{*} \mathrm{E} 16^{*} 14.223$

I16: (F2) [W12] @ ABS $(\mathrm{H} 16-\$ \mathrm{H} \$ 16)$

J16: (F2) 0
K16: (F2) [W12] (\$I\$8+F16)/(F16)

L16: (F2) @LOG $((\$ \mathrm{I} \$ 8+\mathrm{F} 16) /(\mathrm{F} 16))+(\$ \mathrm{~L} \$ 8)^{*}(($ @LOG (F16)))

M16: (F2) [W11] @SQRT(\$I\$8+F16) - @SQRT(F16)

N16: (F2) [W11] (\$N\$8 - H16)

O16: (F2) [W11] (\$O\$8 - H16)

P16: (F2) [W11] (\$P\$8 - H16)

Q16: (F4) [W10] ((\$D\$8*D16*60)/(1000))

R16: (F4) +F16/(\$I\$8+F16)

A17: [W23] 'Pr. B/U TEST

D17: (F4) [W12] 5

E17: (F4) [W10] 1.0417

F17: (F4) $[\mathrm{W} 12]+\$ \mathrm{D} \$ 8^{*} \mathrm{D} 17$

G17: (F2) $[\mathrm{W} 11]+\$ E \$ 8^{*} \mathrm{E} 17$

$\mathrm{H} 17:(\mathrm{F} 2)[\mathrm{W} 12]+\$ \mathrm{E} \$ 8^{*} \mathrm{E} 17^{*} 14.223$

I17: (F2) $[\mathrm{W} 12] @ \mathrm{ABS}(\mathrm{H} 17-\$ \mathrm{H} \$ 16)$

$\mathrm{J} 17:(\mathrm{F} 2)+\mathrm{I} 17+\$ \mathrm{~J} \$ 8$

$\mathrm{K} 17:(\mathrm{F} 2)[\mathrm{W} 12]((\$ \mathrm{I} \$ 8+\mathrm{F} 17) /(\mathrm{F} 17))$

L17: (F2) @LOG $((\$ \mathrm{I} \$ 8+\mathrm{F} 17) /(\mathrm{F} 17))+(\$ \mathrm{~L} \$ 8)^{*}(($ @LOG $(\mathrm{F} 17)))$

M17: (F2) [W11] @SQRT(\$I\$+F17) - @SQRT(F17)

N17: (F2) [W11] (\$N\$8 - H17)

O17: (F2) [W11] (\$O\$8 - H17)

P17: (F2) [W11] (\$P\$8 - H17)

Q17: (F4) $[\mathrm{W} 10]\left(\left(\$ D \$ 8^{*} \mathrm{D} 17^{*} 60\right) /(1000)\right)$

R17: (F4) +F17/(\$I\$8+F17)

A18: [W23] 'Pr. D/D TEST

D18: (F4) [W12] 10

E18: (F4) [W10] 1.056

F18: (F4) $[\mathrm{W} 12]+\$ D \$ 8^{*} \mathrm{D} 18$

G18: (F2) $[\mathrm{W} 11]+\$ E \$ 8^{*} \mathrm{E} 18$

$\mathrm{H} 18:(\mathrm{F} 2)[\mathrm{W} 12]+\$ \mathrm{E} \$ 8^{*} \mathrm{E} 18^{*} 14.223$

I18: (F2) [W12] @ ABS(H18 - \$H\$16)

J18: (F2) +I18+\$J\$8

K18: (F2) [W12] ((\$I\$8+F18)/(F18))

L18: (F2) @LOG $((\$ \mathrm{I} \$ 8+\mathrm{F} 18) /(\mathrm{F} 18))+(\$ \mathrm{~L} \$ 8)^{*}(($ @LOG (F18)))

M18: (F2) [W11] @SQRT(\$I8+F18) - @SQRT(F18)

N18: (F2) [W11] (\$N\$8 - H18)

O18: (F2) [W11] (\$O\$8 - H18)

P18: (F2) [W11] (\$P\$8 - H18)

Q18: (F4) [W10] ((\$D\$8*D18*60)/(1000))

R18: (F4) +F18/(\$I\$8+F18)

A19: [W23] 'PR. FALL OFF TEST (WI WELLS)

D19: (F4) [W12] 15

E19: (F4) [W10] 1.0625

F19: (F4) $[\mathrm{W} 12]+\$ \mathrm{D} \$ 8^{*} \mathrm{D} 19$

G19: (F2) [W11] +\$E $\$ 8^{*} \mathrm{E} 19$

$\mathrm{H} 19:(\mathrm{F} 2)[\mathrm{W} 12]+\$ \mathrm{E} \$ 8^{*} \mathrm{E} 19^{*} 14.223$

I19: (F2) [W12] @ABS(H19 - \$H \$16)

J19: (F2) +I19+\$J\$8

K19: (F2) [W12] ((\$I\$8+F19)/(F19))

L19: (F2) @LOG $((\$ \mathrm{I} \$ 8+\mathrm{F} 19) /(\mathrm{F} 19))+(\$ \mathrm{~L} \$ 8)^{*}(($ @ LOG (F19)))

M19: (F2) [W11] @SQRT(\$I\$8+F19) - @SQRT(F19)

N19: (F2) [W11] (\$N\$8 - H19)

O19: (F2) [W11] (\$O\$8 - H19)

P19: (F2) [W11] (\$P\$8 - H19)

Q19: (F4) [W10] ((\$D\$8*D19*60)/(1000))

R19: (F4) +F19/( $\$$ I $\$ 8+F 19)$ 
A20: [W23] 'PR. FALL OFF TEST

D20: (F4) [W12] 20

E20: (F4) [W10] 1.0698

F20: (F4) $[\mathrm{W} 12]+\$ \mathrm{D} \$ 8^{*} \mathrm{D} 20$

G20: (F2) $[\mathrm{W} 11]+\$ \mathrm{E} \$ 8^{*} \mathrm{E} 20$

$\mathrm{H} 20$ : (F2) [W12] + $\$ \mathrm{E} \$ 8^{*} \mathrm{E} 20^{*} 14.223$

I20: (F2) [W12] @ ABS(H20 - \$H\$16)

J20: (F2) + I20+\$J $\$ 8$

K20: (F2) $[\mathrm{W} 12]((\$ \mathrm{I} \$ 8+\mathrm{F} 20) /(\mathrm{F} 20))$

L20: (F2) @LOG $((\$ I \$ 8+F 20) /(F 20))+(\$ L \$ 8)^{*}((@ L O G$ (F20)))

M20: (F2) [W11] @SQRT(\$I\$8+F20) - @SQRT(F20)

N20: (F2) [W11] ( $\$ \mathrm{~N} \$ 8-\mathrm{H} 20)$

O20: (F2) [W11] (\$O\$8 - H20)

P20: (F2) [W11] (\$P\$8 - H20)

Q20: (F4) [W10] ((\$D\$8*D20*60)/(1000))

R20: (F4) +F20/( $\$$ I $\$ 8+F 20)$

A21: [W23] '(IN - SITU COMBUSTION WELLS)

D21: (F4) [W12] 25

E21: (F4) [W10] 1.0745

F21: (F4) $[\mathrm{W} 12]+\$ \mathrm{D} \$ 8^{*} \mathrm{D} 21$

G21: (F2) [W11] $+\$ \mathrm{E} \$ 8^{*} \mathrm{E} 21$

$\mathrm{H} 21:(\mathrm{F} 2)[\mathrm{W} 12]+\$ \mathrm{E} \$ 8^{*} \mathrm{E} 21^{*} 14.223$

I21: (F2) [W12] @ ABS $(\mathrm{H} 21-\$ \mathrm{H} \$ 16)$

J21: (F2) $+\mathrm{I} 21+\$ \mathrm{~J} \$ 8$

$\mathrm{K} 21:(\mathrm{F} 2)[\mathrm{W} 12]((\$ \mathrm{I} \$ 8+\mathrm{F} 21) /(\mathrm{F} 21))$

L21: (F2) @LOG $((\$ I \$ 8+F 21) /(F 21))+(\$ L \$ 8)^{*}((@ L O G$ $(\mathrm{F} 21)))$

M21: (F2) [W11] @SQRT(\$II8+F21) - @SQRT(F21)

N21: (F2) [W11] ( $\$ \mathrm{~N} \$ 8-\mathrm{H} 21)$

021: (F2) [W11] (\$O\$8 - H21)

P21: (F2) [W11] (\$P\$8 - H21)

Q21: (F4) $[\mathrm{W} 10]\left(\left(\$ D \$ 8^{*} \mathrm{D} 21^{*} 60\right) /(1000)\right)$

R21: (F4) +F21/( $\$$ I $\$ 8+F 21)$

A22: [W23] 'TWO - RATE TEST

D22: (F4) [W12] 30

E22: (F4) [W10] 1.0787

F22: (F4) $[\mathrm{W} 12]+\$ \mathrm{D} \$ 8^{*} \mathrm{D} 22$

G22: (F2) $[\mathrm{W} 11]+\$ \mathrm{E} \$ 8^{*} \mathrm{E} 22$

H22: (F2) [W12] +\$E $\$ 8^{*} \mathrm{E} 22^{*} 14.223$

I22: (F2) [W12] @ ABS $(\mathrm{H} 22-\$ \mathrm{H} \$ 16)$

J22: (F2) + I22+\$J\$8

$\mathrm{K} 22:(\mathrm{F} 2)[\mathrm{W} 12]((\$ \mathrm{I} \$ 8+\mathrm{F} 22) /(\mathrm{F} 22))$

L22: (F2) @LOG $((\$ \mathrm{I} \$ 8+\mathrm{F} 22) /(\mathrm{F} 22))+(\$ \mathrm{~L} \$ 8)^{*}(($ @LOG $(\mathrm{F} 22)))$

M22: (F2) [W11] @SQRT(\$I8+F22) - @SQRT(F22)

N22: (F2) [W11] (\$N\$8- H22)

O22: (F2) [W11] (\$O $\$ 8-\mathrm{H} 22)$

P22: (F2) [W11] (\$P\$8 - H22)

Q22: (F4) [W10] ((\$D\$8*D22*60)/(1000))

R22: (F4) +F22/( $\$$ I $\$ 8+F 22)$

D23: (F4) [W12] 40

E23: (F4) [W10] 1.0795

F23: (F4) $[\mathrm{W} 12]+\$ \mathrm{D} \$ 8^{*} \mathrm{D} 23$

G23: (F2) [W11] $+\$ \mathrm{E} \$ 8^{*} \mathrm{E} 23$

H23: (F2) [W12] + $\$ \mathrm{E} \$ 8^{*} \mathrm{E} 23^{*} 14.223$

I23: (F2) [W12] @ $\mathrm{ABS}(\mathrm{H} 23-\$ \mathrm{H} \$ 16)$

J23: $(\mathrm{F} 2)+\mathrm{I} 23+\$ \mathrm{~J} \$ 8$

K23: (F2) $[\mathrm{W} 12]((\$ \mathrm{I} \$ 8+\mathrm{F} 23) /(\mathrm{F} 23))$

L23: (F2) @LOG $((\$$ I $\$ 8+F 23) /(F 23))+(\$ L \$ 8)^{*}((@ L O G$
$(\mathrm{F} 23)))$

M23: (F2) [W11] @SQRT(\$I\$8+F23) - @SQRT(F23)

N23: (F2) [W11] ( $\$ \mathrm{~N} \$ 8-\mathrm{H} 23)$

O23: (F2) [W11] (\$O \$8 - H23)

P23: (F2) [W11] ( $\$ \mathrm{P} \$ 8-\mathrm{H} 23)$

Q23: (F4) $[\mathrm{W} 10]\left(\left(\$ \mathrm{D} \$ 8^{*} \mathrm{D} 23^{*} 60\right) /(1000)\right)$

R23: (F4) +F23/( $\$$ I $\$ 8+F 23)$

A24: [W23] 'PLOTS:

D24: (F4) [W12] 50

E24: (F4) [W10] 1.0821

F24: (F4) $[\mathrm{W} 12]+\$ D \$ 8^{*} \mathrm{D} 24$

G24: (F2) [W11] $+\$ \mathrm{E} \$ 8^{*} \mathrm{E} 24$

$\mathrm{H} 24:(\mathrm{F} 2)[\mathrm{W} 12]+\$ \mathrm{E} \$ 8^{*} \mathrm{E} 24^{*} 14.223$

I24: (F2) [W12] @ ABS(H24 - \$H\$16)

J24: (F2) +I24+\$J $\$ 8$

K24: (F2) $[\mathrm{W} 12]((\$ \mathrm{I} \$ 8+\mathrm{F} 24) /(\mathrm{F} 24))$

L24: (F2) @LOG $((\$ I \$ 8+F 24) /(F 24))+(\$ L \$ 8)^{*}((@ L O G$ (F24)))

M24: (F2) [W11] @SQRT(\$I\$8+F24) - @SQRT(F24)

N24: (F2) [W11] (\$N\$8 - H24)

O24: (F2) [W11] (\$O \$8 - H24)

P24: (F2) [W11] (\$P\$8 - H24)

Q24: (F4) [W10] ((\$D\$8*D24*60)/(1000))

R24: (F4) +F24/( $\$$ I $\$ 8+F 24)$

D25: (F4) [W12] 70

E25: (F4) [W10] 1.095

F25: (F4) $[\mathrm{W} 12]+\$ D \$ 8^{*} \mathrm{D} 25$

G25: (F2) [W11] $+\$ E \$ 8^{*} \mathrm{E} 25$

$\mathrm{H} 25:(\mathrm{F} 2)[\mathrm{W} 12]+\$ \mathrm{E} \$ 8^{*} \mathrm{E} 25^{*} 14.223$

I25: (F2) [W12] @ $\mathrm{ABS}(\mathrm{H} 25-\$ \mathrm{H} \$ 16)$

J25: (F2) +I $25+\$ J \$ 8$

K25: (F2) [W12] $((\$ \mathrm{I} \$ 8+\mathrm{F} 25) /(\mathrm{F} 25))$

L25: (F2) @LOG $((\$ 1 \$ 8+F 25) /(F 25))+(\$ L \$ 8)^{*}((@ L O G$ $(\mathrm{F} 25))$ )

M25: (F2) [W11] @SQRT(\$I\$8+F25) - @SQRT(F25)

N25: (F2) [W11] (\$N\$8 - H25)

O25: (F2) [W11] (\$O\$8 - H25)

P25: (F2) [W11] (\$P \$8 - H25)

Q25: (F4) [W10] ((\$D\$8*D25*60)/(1000))

R25: (F4) +F25/(\$I\$8+F25)

A26: [W23] 'ZERO TIME PR. ERROR

D26: (F4) [W12] 80

E26: (F4) [W10] 1.0988

F26: (F4) $[\mathrm{W} 12]+\$ \mathrm{D} \$ 8^{*} \mathrm{D} 26$

G26: (F2) $[\mathrm{W} 11]+\$ \mathrm{E} \$ 8^{*} \mathrm{E} 26$

$\mathrm{H} 26:(\mathrm{F} 2)[\mathrm{W} 12]+\$ \mathrm{E} \$ 8^{*} \mathrm{E} 26^{*} 14.223$

I26: (F2) [W12] @ ABS(H26 - \$H\$16)

J26: (F2) +I26+\$J \$8

K26: (F2) [W12] $((\$ 1 \$ 8+F 26) /(F 26))$

L26: (F2) @LOG $((\$ 1 \$ 8+F 26) /(F 26))+(\$ L \$ 8)^{*}((@ L O G$ $(\mathrm{F} 26))$ )

M26: (F2) [W11] @SQRT(\$I\$8+F26) - @SQRT(F26)

N26: (F2) [W11] (\$N\$8 - H26)

O26: (F2) [W11] (\$O \$8 - H26)

P26: (F2) [W11] (\$P\$8 - H26)

Q26: (F4) [W10] ((\$D\$8*D26*60)/(1000))

R26: (F4) +F26/( $\$$ I $\$ 8+F 26)$

A27: [W23] 'LIN. ANALYSIS

D27: (F4) [W12] 90

E27: (F4) [W10] 1.1021 
F27: (F4) $[\mathrm{W} 12]+\$ \mathrm{D} \$ 8^{*} \mathrm{D} 27$

G27: (F2) $[\mathrm{W} 11]+\$ \mathrm{E} \$ 8^{*} \mathrm{E} 27$

$\mathrm{H} 27:(\mathrm{F} 2)[\mathrm{W} 12]+\$ \mathrm{E} \$ 8^{*} \mathrm{E} 27^{*} 14.223$

I27: (F2) [W12] @ $\mathrm{ABS}(\mathrm{H} 27-\$ \mathrm{H} \$ 16)$

J27: $(\mathrm{F} 2)+\mathrm{I} 27+\$ \mathrm{~J} \$ 8$

K27: (F2) $[\mathrm{W} 12]((\$ \mathrm{I} \$ 8+\mathrm{F} 27) /(\mathrm{F} 27))$

L27: (F2) @LOG $((\$$ I $\$ 8+F 27) /(\mathrm{F} 27))+(\$ L \$ 8)^{*}((@ \mathrm{LOG}$ (F27)))

M27: (F2) [W11] @SQRT(\$I\$8+F27) - @SQRT(F27)

N27: (F2) [W11] (\$N\$8 - H27)

O27: (F2) [W11] (\$O\$8 - H27)

P27: (F2) [W11] ( $\$ \mathrm{P} \$ 8-\mathrm{H} 27)$

Q27: (F4) [W10] ((\$D\$8*D27*60)/(1000))

R27: (F4) +F27/( $\$$ I $\$ 8+F 27)$

A28: [W23] 'DIAGNOSTIC

D28: (F4) [W12] 100

E28: (F4) [W10] 1.105

F28: (F4) [W12] + $\$ \mathrm{D} \$ 8^{*} \mathrm{D} 28$

G28: (F2) $[\mathrm{W} 11]+\$ \mathrm{E} \$ 8^{*} \mathrm{E} 28$

H28: (F2) $[\mathrm{W} 12]+\$ \mathrm{E} \$ 8^{*} \mathrm{E} 28^{*} 14.223$

I28: (F2) [W12] @ ABS $(\mathrm{H} 28-\$ \mathrm{H} \$ 16)$

J28: $(\mathrm{F} 2)+\mathrm{I} 28+\$ \mathrm{~J} \$ 8$

K28: (F2) [W12] ((\$I\$8+F28)/(F28))

L28: (F2) @LOG $((\$ \mathrm{I} \$ 8+\mathrm{F} 28) /(\mathrm{F} 28))+(\$ \mathrm{~L} \$ 8)^{*}(($ @LOG (F28)) )

M28: (F2) [W11] @SQRT(\$I8+F28) - @SQRT(F28)

N28: (F2) [W11] ( $\$ \mathrm{~N} \$ 8-\mathrm{H} 28)$

O28: (F2) [W11] ( $\$ \mathrm{O} \$ 8$ - H28)

P28: (F2) [W11] (\$P\$8 - H28)

Q28: (F4) [W10] ((\$D\$8*D28*60)/(1000))

R28: (F4) +F28/( $\$$ I $\$ 8+F 28)$

A29: [W23] 'MDH

D29: (F4) [W12] 110

E29: (F4) [W10] 1.1075

F29: (F4) $[\mathrm{W} 12]+\$ \mathrm{D} \$ 8^{*} \mathrm{D} 29$

G29: (F2) $[\mathrm{W} 11]+\$ \mathrm{E} \$ 8^{*} \mathrm{E} 29$

H29: (F2) [W12] + $\$ E \$ 8^{*} \mathrm{E} 29^{*} 14.223$

I29: (F2) [W12] @ ABS $(\mathrm{H} 29$ - $\$ \mathrm{H} \$ 16)$

J29: (F2) +I29+\$J\$8

K29: (F2) [W12] ((\$I\$8+F29)/(F29))

L29: (F2) @LOG $((\$ \mathrm{I} \$ 8+\mathrm{F} 29) /(\mathrm{F} 29))+(\$ \mathrm{~L} \$ 8)^{*}(($ @LOG (F29)))

M29: (F2) [W11] @SQRT(\$I \$8+F29) - @SQRT(F29)

N29: (F2) [W11] ( $\$ \mathrm{~N} \$ 8-\mathrm{H} 29)$

O29: (F2) [W11] (\$O\$8 - H29)

P29: (F2) [W11] (\$P\$8 - H29)

Q29: (F4) [W10] ((\$D\$8* D29*60)/(1000))

R29: (F4) +F29/( $\$$ I $\$ 8+F 29)$

A30: [W23] 'HORNER

D30: (F4) [W12] 120

E30: (F4) [W10] 1.108

F30: (F4) $[\mathrm{W} 12]+\$ \mathrm{D} \$ 8^{*} \mathrm{D} 30$

G30: (F2) $[\mathrm{W} 11]+\$ \mathrm{E} \$ 8^{*} \mathrm{E} 30$

H30: (F2) [W12] +\$E $\$ 8^{*} \mathrm{E} 30^{*} 14.223$

I30: (F2) [W12] @ ABS $(\mathrm{H} 30-\$ \mathrm{H} \$ 16)$

J30: (F2) $+\mathrm{I} 30+\$ \mathrm{~J} \$ 8$

K30: (F2) [W12] $((\$ I \$ 8+F 30) /(F 30))$

L30: (F2) @LOG $((\$ \mathrm{I} \$ 8+\mathrm{F} 30) /(\mathrm{F} 30))+(\$ \mathrm{~L} \$ 8)^{*}(($ @LOG (F30)))

M30: (F2) [W11] @SQRT(\$I8+F30) - @SQRT(F30)
N30: (F2) [W11] (\$N\$8 - H30)

O30: (F2) [W11] (\$O\$8 - H30)

P30: (F2) [W11] (\$P\$8 - H30)

Q30: (F4) [W10] ((\$D\$8*D30*60)/(1000))

R30: (F4) +F30/(\$I\$8+F30)

A31: [W23] 'MUSKAT

D31: (F4) [W12] 140

E31: (F4) [W10] 1.111

F31: (F4) $[\mathrm{W} 12]+\$ \mathrm{D} \$ 8^{*} \mathrm{D} 31$

G31: (F2) $[\mathrm{W} 11]+\$ \mathrm{E} \$ 8^{*} \mathrm{E} 31$

$\mathrm{H} 31:(\mathrm{F} 2)[\mathrm{W} 12]+\$ \mathrm{E} \$ 8^{*} \mathrm{E} 31^{*} 14.223$

I31: (F2) [W12] @ABS(H31 - \$H\$16)

J31: (F2) $+\mathrm{I} 31+\$ \mathrm{~J} \$ 8$

K31: (F2) [W12] $((\$ I \$ 8+F 31) /(F 31))$

L31: (F2) @LOG $((\$$ I $\$ 8+F 31) /(F 31))+(\$ L \$ 8)^{*}((@$ LOG $(\mathrm{F} 31))$

M31: (F2) [W11] @SQRT(\$I8+F31) - @SQRT(F31)

N31: (F2) [W11] (\$N\$8 - H31)

O31: (F2) [W11] (\$O\$8 - H31)

P31: (F2) [W11] (\$P\$8 - H31)

Q31: (F4) [W10] ((\$D\$8*D31*60)/(1000))

R31: (F4) +F31/(\$I\$8+F31)

A32: [W23] 'SEMI STEADY STAE ANALYSIS

D32: (F4) [W12] 200

E32: (F4) [W10] 1.1135

F32: (F4) $[\mathrm{W} 12]+\$ \mathrm{D} \$ 8^{*} \mathrm{D} 32$

G32: (F2) $[\mathrm{W} 11]+\$ \mathrm{E} \$ 8^{*} \mathrm{E} 32$

$\mathrm{H} 32:(\mathrm{F} 2)[\mathrm{W} 12]+\$ \mathrm{E} \$ 8^{*} \mathrm{E} 32^{*} 14.223$

I32: (F2) [W12] @ ABS $(\mathrm{H} 32-\$ \mathrm{H} \$ 16)$

J32: (F2) $+\mathrm{I} 32+\$ \mathrm{~J} \$ 8$

K32: (F2) $[\mathrm{W} 12]((\$ \mathrm{I} \$ 8+\mathrm{F} 32) /(\mathrm{F} 32))$

L32: (F2) @LOG $((\$$ I $\$ 8+F 32) /(F 32))+(\$ L \$ 8)^{*}(($ @LOG (F32)))

M32: (F2) [W11] @SQRT(\$I\$8+F32) - @SQRT(F32)

N32: (F2) [W11] (\$N\$8 - H32)

O32: (F2) [W11] (\$O\$8 - H32)

P32: (F2) [W11] (\$P\$8 - H32)

Q32: (F4) [W10] ((\$D\$8*D32*60)/(1000))

R32: (F4) +F32/( $\$$ I $\$ 8+F 32)$

A33: [W23] 'PLOT FOR WI WELL

D33: (F4) [W12] 250

E33: (F4) [W10] 1.1145

F33: (F4) $[\mathrm{W} 12]+\$ \mathrm{D} \$ 8^{*} \mathrm{D} 33$

G33: (F2) $[\mathrm{W} 11]+\$ \mathrm{E} \$ 8^{*} \mathrm{E} 33$

H33: (F2) $[\mathrm{W} 12]+\$ \mathrm{E} \$ 8^{*} \mathrm{E} 33^{*} 14.223$

I33: (F2) [W12] @ ABS $(\mathrm{H} 33-\$ \mathrm{H} \$ 16)$

J33: $(\mathrm{F} 2)+\mathrm{I} 33+\$ \mathrm{~J} \$ 8$

K33: (F2) [W12] ((\$I\$8+F33)/(F33))

L33: (F2) @LOG $((\$$ I $\$ 8+F 33) /(F 33))+(\$ L \$ 8) *((@ L O G$ (F33)))

M33: (F2) [W11] @SQRT(\$I\$8+F33) - @SQRT(F33)

N33: (F2) [W11] (\$N\$8 - H33)

O33: (F2) [W11] (\$O\$8 - H33)

P33: (F2) [W11] (\$P\$8 - H33)

Q33: (F4) [W10] ((\$D\$8*D33*60)/(1000))

R33: (F4) +F33/(\$I\$8+F33)

A34: [W23] 'TWO - RATE TEST PLOT

D34: (F4) [W12] 300

E34: (F4) [W10] 1.116

F34: (F4) [W12] +\$D\$8*D34 
G34: (F2) $[\mathrm{W} 11]+\$ \mathrm{E} \$ 8^{*} \mathrm{E} 34$

H34: (F2) [W12] +\$E $\$ 8^{*} \mathrm{E} 34^{*} 14.223$

I34: (F2) [W12] @ ABS(H34-\$H\$16)

J34: $(\mathrm{F} 2)+\mathrm{I} 34+\$ \mathrm{~J} \$ 8$

K34: (F2) [W12] $((\$ \mathrm{I} \$ 8+\mathrm{F} 34) /(\mathrm{F} 34))$

L34: (F2) @LOG $((\$$ I $\$ 8+F 34) /(F 34))+(\$ L \$ 8)^{*}((@ L O G$ (F34)))

M34: (F2) [W11] @SQRT(\$I\$8+F34) - @SQRT(F34)

N34: (F2) [W11] (\$N\$8 - H34)

034: (F2) [W11] (\$O\$8 - H34)

P34: (F2) [W11] (\$P\$8 - H34)

Q34: (F4) [W10] ((\$D\$8*D34*60)/(1000))

R34: (F4) +F34/( $\$$ I $\$ 8+F 34)$

A35: [W23] 'WRB PLOT

D35: (F4) [W12] 400

E35: (F4) [W10] 1.117

F35: (F4) $[\mathrm{W} 12]+\$ \mathrm{D} \$ 8^{*} \mathrm{D} 35$

G35: (F2) [W11] + $\$ \mathrm{E} \$ 8^{*} \mathrm{E} 35$

H35: (F2) [W12] + $\$ \mathrm{E} \$ 8^{*} \mathrm{E} 35^{*} 14.223$

I35: (F2) [W12] @ ABS $(\mathrm{H} 35-\$ \mathrm{H} \$ 16)$

J35: $(\mathrm{F} 2)+\mathrm{I} 35+\$ \mathrm{~J} \$ 8$

K35: (F2) $[\mathrm{W} 12]((\$ \mathrm{I} \$ 8+\mathrm{F} 35) /(\mathrm{F} 35))$

L35: (F2) @LOG $((\$ \mathrm{I} \$ 8+\mathrm{F} 35) /(\mathrm{F} 35))+(\$ \mathrm{~L} \$ 8)^{*}(($ @ LOG $(\mathrm{F} 35)))$

M35: (F2) [W11] @SQRT(\$I8+F35) - @SQRT(F35)

N35: (F2) [W11] (\$N\$8 - H35)

O35: (F2) [W11] (\$O \$8 - H35)

P35: (F2) [W11] (\$P\$8 - H35)

Q35: (F4) [W10] ((\$D\$8*D35*60)/(1000))

R35: (F4) +F35/( $\$$ I $\$ 8+F 35)$

D36: (F4) [W12] 500

E36: (F4) [W10] 1.118

F36: (F4) $[\mathrm{W} 12]+\$ D \$ 8^{*} \mathrm{D} 36$

G36: (F2) [W11] + $\$ \mathrm{E} \$ 8^{*} \mathrm{E} 36$

H36: (F2) $[\mathrm{W} 12]+\$ \mathrm{E} \$ 8^{*} \mathrm{E} 36^{*} 14.223$

I36: (F2) [W12] @ $\mathrm{ABS}(\mathrm{H} 36-\$ \mathrm{H} \$ 16)$

J36: $(\mathrm{F} 2)+\mathrm{I} 36+\$ \mathrm{~J} \$ 8$

K36: (F2) [W12] ((\$I\$8+F36)/(F36))

L36: (F2) @LOG $((\$ 1 \$ 8+F 36) /(\mathrm{F} 36))+(\$ L \$ 8)^{*}((@ \mathrm{LOG}$ (F36)) )

M36: (F2) [W11] @SQRT(\$I\$8+F36) - @SQRT(F36)

N36: (F2) [W11] (\$N\$8 - H36)

O36: (F2) [W11] (\$O\$8- H36)

P36: (F2) [W11] (\$P\$8 - H36)

Q36: (F4) [W10] ((\$D\$8*D36*60)/(1000))

R36: (F4) +F36/( $\$$ I $\$ 8+F 36)$

D37: (F4) [W12] 700

E37: (F4) [W10] 1.119

F37: (F4) $[\mathrm{W} 12]+\$ \mathrm{D} \$ 8^{*} \mathrm{D} 37$

G37: (F2) $[\mathrm{W} 11]+\$ \mathrm{E} \$ 8^{*} \mathrm{E} 37$

$\mathrm{H} 37:(\mathrm{F} 2)[\mathrm{W} 12]+\$ \mathrm{E} \$ 8^{*} \mathrm{E} 37^{*} 14.223$

I37: (F2) [W12] @ ABS $(\mathrm{H} 37-\$ \mathrm{H} \$ 16)$

J37: $(\mathrm{F} 2)+\mathrm{I} 37+\$ \mathrm{~J} \$ 8$

K37: (F2) $[\mathrm{W} 12]((\$ \mathrm{I} \$ 8+\mathrm{F} 37) /(\mathrm{F} 37))$

L37: (F2) @LOG $((\$$ I $\$ 8+F 37) /(F 37))+(\$ L \$ 8)^{*}((@$ LOG $(\mathrm{F} 37)))$

M37: (F2) [W11] @SQRT(\$I8+F37) - @SQRT(F37)

N37: (F2) [W11] (\$N\$8 - H37)

O37: (F2) [W11] (\$O\$8 - H37)

P37: (F2) [W11] (\$P\$8 - H37)
Q37: (F4) [W10] ((\$D\$8*D37*60)/(1000))

R37: (F4) +F37/(\$I8+F37)

D38: (F4) [W12] 900

E38: (F4) [W10] 1.1205

F38: (F4) $[\mathrm{W} 12]+\$ D \$ 8^{*} \mathrm{D} 38$

G38: (F2) $[\mathrm{W} 11]+\$ \mathrm{E} \$ 8^{*} \mathrm{E} 38$

H38: (F2) [W12] + $\$ \mathrm{E} \$ 8^{*} \mathrm{E} 38^{*} 14.223$

I38: (F2) [W12] @ ABS(H38 - \$H \$16)

J38: $(\mathrm{F} 2)+\mathrm{I} 38+\$ \mathrm{~J} \$ 8$

K38: (F2) [W12] ((\$I\$8+F38)/(F38))

L38: (F2) @LOG $((\$ \mathrm{I} \$ 8+\mathrm{F} 38) /(\mathrm{F} 38))+(\$ \mathrm{~L} \$ 8)^{*}(($ @LOG $(\mathrm{F} 38)))$

M38: (F2) [W11] @SQRT(\$I \$8+F38) - @SQRT(F38)

N38: (F2) [W11] ( $\$ \mathrm{~N} \$ 8-\mathrm{H} 38)$

O38: (F2) [W11] (\$O\$8 - H38)

P38: (F2) [W11] (\$P\$8 - H38)

Q38: (F4) [W10] ((\$D\$8*D38*60)/(1000))

R38: (F4) +F38/(\$I\$8+F38)

D39: (F4) [W12] 1100

E39: (F4) [W10] 1.121

F39: (F4) $[\mathrm{W} 12]+\$ \mathrm{D} \$ 8^{*} \mathrm{D} 39$

G39: (F2) $[\mathrm{W} 11]+\$ \mathrm{E} \$ 8^{*} \mathrm{E} 39$

H39: (F2) [W12] +\$E\$8*E39*14.223

I39: (F2) [W12] @ ABS(H39-\$H\$16)

J39: (F2) +I39+\$J\$8

K39: (F2) [W12] ((\$I\$8+F39)/(F39))

L39: (F2) @LOG $((\$$ I $\$ 8+F 39) /($ F39 $))+(\$ L \$ 8)^{*}((@ L O G$ (F39)))

M39: (F2) [W11] @SQRT(\$I8+F39) - @SQRT(F39)

N39: (F2) [W11] (\$N\$8 - H39)

O39: (F2) [W11] (\$O\$8 - H39)

P39: (F2) [W11] (\$P\$8 - H39)

Q39: (F4) [W10] ((\$D\$8*D39*60)/(1000))

R39: (F4) +F39/( $\$$ I $\$ 8+F 39)$

D40: (F4) [W12] 1500

E40: (F4) [W10] 1.1225

F40: (F4) $[\mathrm{W} 12]+\$ \mathrm{D} \$ 8^{*} \mathrm{D} 40$

G40: (F2) $[\mathrm{W} 11]+\$ \mathrm{E} \$ 8^{*} \mathrm{E} 40$

$\mathrm{H} 40:(\mathrm{F} 2)[\mathrm{W} 12]+\$ \mathrm{E} \$ 8^{*} \mathrm{E} 40^{*} 14.223$

I40: (F2) [W12] @ ABS $(\mathrm{H} 40-\$ \mathrm{H} \$ 16)$

J40: (F2) $+\mathrm{I} 40+\$ \mathrm{~J} \$ 8$

$\mathrm{K} 40:(\mathrm{F} 2)[\mathrm{W} 12]((\$ \mathrm{I} \$ 8+\mathrm{F} 40) /(\mathrm{F} 40))$

L40: (F2) @LOG $((\$ \mathrm{I} \$ 8+\mathrm{F} 40) /(\mathrm{F} 40))+(\$ \mathrm{~L} \$ 8) *((@ \mathrm{LOG}$ $(\mathrm{F} 40)))$

M40: (F2) [W11] @SQRT(\$I\$8+F40) - @SQRT(F40)

$\mathrm{N} 40:(\mathrm{F} 2)[\mathrm{W} 11](\$ \mathrm{~N} \$ 8-\mathrm{H} 40)$

O40: (F2) [W11] (\$O\$8 - H40)

P40: (F2) [W11] (\$P\$8 - H40)

Q40: (F4) [W10] ((\$D\$8*D40*60)/(1000))

R40: (F4) +F40/( $\$$ I $\$ 8+F 40)$

D41: (F4) [W12] 1800

E41: (F4) [W10] 1.1235

F41: (F4) [W12] +\$D\$8*D41

G41: (F2) [W11] $+\$ \mathrm{E} \$ 8^{*} \mathrm{E} 41$

$\mathrm{H} 41:$ (F2) $[\mathrm{W} 12]+\$ \mathrm{E} \$ 8^{*} \mathrm{E} 41^{*} 14.223$

I41: (F2) [W12] @ ABS $(\mathrm{H} 41-\$ \mathrm{H} \$ 16)$

$\mathrm{J} 41:(\mathrm{F} 2)+\mathrm{I} 41+\$ \mathrm{~J} \$ 8$

K41: (F2) [W12] ((\$I\$8+F41)/(F41))

L41: (F2) @LOG $((\$ I \$ 8+F 41) /(F 41))+(\$ L \$ 8)^{*}((@ L O G$ $(\mathrm{F} 41)))$ 
M41: (F2) [W11] @SQRT(\$I\$8+F41) - @SQRT(F41)

N41: (F2) [W11] (\$N\$8 - H41)

O41: (F2) [W11] (\$O\$8 - H41)

P41: (F2) [W11] (\$P\$8 - H41)

Q41: (F4) [W10] ((\$D\$8*D41*60)/(1000))

R41: (F4) +F41/ $(\$ \mathrm{I} \$ 8+\mathrm{F} 41)$

D42: (F4) [W12] 2083

E42: (F4) [W10] 1.1235

F42: (F4) [W12] $+\$ D \$ 8^{*} \mathrm{D} 42$

G42: (F2) $[\mathrm{W} 11]+\$ \mathrm{E} \$ 8^{*} \mathrm{E} 42$

$\mathrm{H} 42:(\mathrm{F} 2)[\mathrm{W} 12]+\$ \mathrm{E} \$ 8^{*} \mathrm{E} 42^{*} 14.223$

I42: (F2) [W12] @ ABS(H42 - \$H\$16)

$\mathrm{J} 42:(\mathrm{F} 2)+\mathrm{I} 42+\$ \mathrm{~J} \$ 8$

K42: (F2) [W12] $((\$ 1 \$ 8+F 42) /(\mathrm{F} 42))$

L42: (F2) @LOG $((\$ \mathrm{I} \$ 8+\mathrm{F} 42) /(\mathrm{F} 42))+(\$ \mathrm{~L} \$ 8)^{*}(($ @ LOG (F42))

M42: (F2) [W11] @SQRT(\$I\$8+F42) - @SQRT(F42)

N42: (F2) [W11] ( $\$ \mathrm{~N} \$ 8$ - H42)

O42: (F2) [W11] (\$O\$8 - H42)

P42: (F2) [W11] (\$P\$8 - H42)

Q42: (F4) $[\mathrm{W} 10]\left(\left(\$ \mathrm{D} \$ 8^{*} \mathrm{D} 42^{*} 60\right) /(1000)\right)$

R42: (F4) +F42/(\$I $\$ 8+F 42)$

D43: (F4) [W12] 2916

E43: (F4) [W10] 1.127

F43: (F4) $[\mathrm{W} 12]+\$ \mathrm{D} \$ 8^{*} \mathrm{D} 43$

G43: (F2) [W11] $+\$ \mathrm{E} \$ 8^{*} \mathrm{E} 43$

H43: (F2) $[\mathrm{W} 12]+\$ E \$ 8^{*} \mathrm{E} 43^{*} 14.223$

I43: (F2) [W12] @ ABS $(\mathrm{H} 43-\$ \mathrm{H} \$ 16)$

J43: (F2) +I43+\$J\$8

K43: (F2) [W12] $((\$ \mathrm{I} \$ 8+\mathrm{F} 43) /(\mathrm{F} 43))$

L43: (F2)@LOG $((\$ \mathrm{I} \$ 8+\mathrm{F} 43) /(\mathrm{F} 43))+(\$ \mathrm{~L} \$ 8)^{*}(($ @ LOG (F43)))

M43: (F2) [W11] @SQRT(\$I\$8+F43) - @SQRT(F43)

N43: (F2) [W11] (\$N\$8 - H43)

O43: (F2) [W11] (\$O\$8 - H43)

P43: (F2) [W11] (\$P \$8 - H43)

Q43: (F4) $[\mathrm{W} 10]\left(\left(\$ \mathrm{D} \$ 8^{*} \mathrm{D} 43^{*} 60\right) /(1000)\right)$

R43: (F4) +F43/( $\$$ I\$8+F43)

D44: (F4) [W12] 3750

E44: (F4) [W10] 1.128

F44: (F4) $[\mathrm{W} 12]+\$ D \$ 8^{*} \mathrm{D} 44$

G44: (F2) $[\mathrm{W} 11]+\$ \mathrm{E} \$ 8^{*} \mathrm{E} 44$

$\mathrm{H} 44:(\mathrm{F} 2)[\mathrm{W} 12]+\$ \mathrm{E} \$ 8^{*} \mathrm{E} 44^{*} 14.223$

I44: (F2) [W12] @ ABS(H44 - \$H \$16)

J44: (F2) +I44+\$J $\$ 8$

K44: (F2) $[\mathrm{W} 12]((\$ \mathrm{I} \$ 8+\mathrm{F} 44) /(\mathrm{F} 44))$

L44: (F2) @LOG $((\$ 1 \$ 8+F 44) /(F 44))+(\$ L \$ 8)^{*}((@ L O G$ $(\mathrm{F} 44)))$

M44: (F2) [W11] @SQRT(\$I\$8+F44) - @SQRT(F44)

N44: (F2) [W11] (\$N\$8 - H44)

O44: (F2) [W11] (\$O\$8 - H44)

P44: (F2) [W11] (\$P\$8 - H44)

Q44: (F4) [W10] $\left(\left(\$ D \$ 8^{*} \mathrm{D} 44^{*} 60\right) /(1000)\right)$

R44: (F4) +F44/(\$I\$8+F44)

D45: (F4) [W12] 4166

E45: (F4) [W10] 1.128

F45: (F4) $[\mathrm{W} 12]+\$ \mathrm{D} \$ 8^{*} \mathrm{D} 45$

G45: (F2) $[\mathrm{W} 11]+\$ \mathrm{E} \$ 8^{*} \mathrm{E} 45$

H45: (F2) $[\mathrm{W} 12]+\$ E \$ 8^{*} \mathrm{E} 45^{*} 14.223$

I45: (F2) [W12]@ ABS(H45 - \$H \$16)
J45: (F2) +I45+\$J\$8

K45: (F2) [W12] $((\$ 1 \$ 8+\mathrm{F} 45) /(\mathrm{F} 45))$

L45: (F2) @LOG $((\$ I \$ 8+F 45) /(F 45))+(\$ L \$ 8)^{*}((@ L O G$ (F45)))

M45: (F2) [W11] @SQRT(\$I\$8+F45) - @SQRT(F45)

N45: (F2) [W11] (\$N\$8 - H45)

O45: (F2) [W11] (\$O\$8 - H45)

P45: (F2) [W11] (\$P\$8 - H45)

Q45: (F4) $[\mathrm{W} 10]\left(\left(\$ \mathrm{D} \$ 8^{*} \mathrm{D} 45^{*} 60\right) /(1000)\right)$

$\mathrm{R} 45:(\mathrm{F} 4)+\mathrm{F} 45 /(\$ \mathrm{I} \$ 8+\mathrm{F} 45)$

D46: (F4) [W12] 4219

E46: (F4) [W10] 1.128

F46: (F4) $[\mathrm{W} 12]+\$ D \$ 8^{*} \mathrm{D} 46$

G46: (F2) $[\mathrm{W} 11]+\$ \mathrm{E} \$ 8^{*} \mathrm{E} 46$

$\mathrm{H} 46$ : (F2) $[\mathrm{W} 12]+\$ \mathrm{E} \$ 8^{*} \mathrm{E} 46^{*} 14.223$

I46: (F2) [W12] @ ABS $(\mathrm{H} 46-\$ \mathrm{H} \$ 16)$

J46: (F2) +I46+\$J $\$ 8$

K46: (F2) $[\mathrm{W} 12]((\$ \mathrm{I} \$ 8+\mathrm{F} 46) /(\mathrm{F} 46))$

L46: (F2) @LOG $((\$ I \$ 8+F 46) /(F 46))+(\$ L \$ 8)^{*}((@ L O G$ $(\mathrm{F} 46)))$

M46: (F2) [W11] @SQRT(\$I\$8+F46) - @SQRT(F46)

N46: (F2) [W11] (\$N\$8 - H46)

O46: (F2) [W11] (\$O\$8 - H46)

P46: (F2) [W11] ( $\$ \mathrm{P} \$ 8$ - H46)

Q46: (F4) [W10] ((\$D\$8*D46*60)/(1000))

R46: (F4) +F46/(\$I\$8+F46)

A51: [W23] 'INFLUX - STUDY:

F51: [W12] 'Pi(psi)

G51: [W11] ^ $\mathrm{Po}(\mathrm{psi})$

F52: [W12] 1750

G52: [W11] 1067.76

D55: (F2) [W12] ^ D

E55: (F2) [W10] ${ }^{\wedge} \mathrm{E}$

F55: (F2) [W12] ^ $\mathrm{F}$

G55: (F2) [W11] ^ $\mathrm{G}$

$\mathrm{H} 55$ : [W12] ^ $\mathrm{H}$

I55: [W12] ^ I

J55: ^ J

K55: [W12] ^ $\mathrm{K}$

L55: ${ }^{\wedge} \mathrm{L}$

M55: [W11] ^M

D56: (F2) [W12] ^ DEL. TIME

E56: (F2) [W10] ^ PRESSURE

F56: (F2) [W12] ^ DEL. TIME

G56: (F2) [W11] ^ PRESSURE

H56: [W12] ^ PRESSURE

I56: [W12] ^ DEL - P

J56: ^ DEL - P/DEL - T

K56: [W12] $\wedge$ DEL - P/DEL - T

L56: ^ TIME

M56: [W11] ^ (Pi - Pw $) /$

D57: (F2) [W12] ^ div.

E57: (F2) [W10] $\mathrm{mm}$

F57: (F2) [W12] ^ $\mathrm{hr}$

G57: (F2) [W11] ${ }^{\wedge} \mathrm{kg} / \mathrm{cm} 2$

H57: [W12] ^psi

I57: [W12] ^ psi

J57: ^ $\mathrm{psi} / 1 \mathrm{hr}$

K57: [W12] ${ }^{-p s i / 2 h r}$

L57: ' minute 
M57: [W11] $(\mathrm{Pi}-\mathrm{Po})$

D59: (F4) [W12] 0

E59: (F4) [W10] 0.9366

F59: (F4) $[\mathrm{W} 12]+\$ \mathrm{D} \$ 8^{*} \mathrm{D} 59$

G59: (F2) [W11] $+\$ \mathrm{E} \$ 8^{*} \mathrm{E} 59$

H59: (F2) [W12] + \$E $\$ 8^{*} \mathrm{E} 59^{*} 14.223$

I59: (F2) [W12] @ ABS(H59 - \$H\$59)

L59: (F2) (D59* $\$ D \$ 8)^{*} 60$

M59: (F2) [W11] (\$F $\$ 52-\mathrm{H} 59) /(\$ F \$ 52-\$ G \$ 52)$

D60: (F4) $[\mathrm{W} 12]+\mathrm{D} 59+69.44$

E60: (F4) [W10] 0.9656

F60: (F4) $[\mathrm{W} 12]+\$ \mathrm{D} \$ 8^{*} \mathrm{D} 60$

G60: (F2) $[\mathrm{W} 11]+\$ \mathrm{E} \$ 8^{*} \mathrm{E} 60$

$\mathrm{H} 60:(\mathrm{F} 2)[\mathrm{W} 12]+\$ \mathrm{E} \$ 8^{*} \mathrm{E} 60^{*} 14.223$

I60: (F2) [W12] @ ABS $(\mathrm{H} 60$ - \$H\$59)

J60: (F2) (H61 - H60)

L60: (F2) (D60*\$D $\$ 8)^{*} 60$

M60: (F2) [W11] (\$F\$52 - H60)/( $\$ F \$ 52$ - \$G\$52)

$\mathrm{D} 61:(\mathrm{F} 4)[\mathrm{W} 12]+\mathrm{D} 60+69.44$

E61: (F4) [W10] 0.9917

F61: (F4) $[\mathrm{W} 12]+\$ D \$ 8^{*} \mathrm{D} 61$

G61: (F2) [W11] + $\$ \mathrm{E} \$ 8^{*} \mathrm{E} 61$

H61: (F2) [W12] +\$E $\$ 8^{*} \mathrm{E} 61^{*} 14.223$

I61: (F2) [W12] @ ABS(H61 - \$H\$59)

J61: (F2) (H62 - H61)

K61: (F2) [W12] (H61 - H59)

L61: (F2) (D61*\$D $\$ 8)^{*} 60$

M61: (F2) [W11] (\$F $\$ 52-\mathrm{H} 61) /(\$ F \$ 52-\$ G \$ 52)$

D62: (F4) $[\mathrm{W} 12]+\mathrm{D} 61+69.44$

E62: (F4) [W10] 1.0149

F62: (F4) [W12] $+\$ D \$ 8^{*} \mathrm{D} 62$

G62: (F2) [W11] + $\$ \mathrm{E} \$ 8^{*} \mathrm{E} 62$

$\mathrm{H} 62:(\mathrm{F} 2)[\mathrm{W} 12]+\$ \mathrm{E} \$ 8^{*} \mathrm{E} 62^{*} 14.223$

I62: (F2) [W12] @ ABS(H62 - \$H\$59)

J62: (F2) (H63 - H62)

K62: (F2) [W12] (H62 - H60)

L62: (F2) (D62*\$D \$8)*60

M62: (F2) [W11] (\$F $\$ 52-\mathrm{H} 62) /(\$ F \$ 52$ - $\$ \mathrm{G} \$ 52)$

D63: (F4) $[\mathrm{W} 12]+\mathrm{D} 62+69.44$

E63: (F4) [W10] 1.0356

F63: (F4) $[\mathrm{W} 12]+\$ D \$ 8^{*} \mathrm{D} 63$

G63: (F2) $[\mathrm{W} 11]+\$ \mathrm{E} \$ 8^{*} \mathrm{E} 63$

H63: (F2) $[\mathrm{W} 12]+\$ \mathrm{E} \$ 8^{*} \mathrm{E} 63^{*} 14.223$

I63: (F2) [W12] @ $\mathrm{ABS}(\mathrm{H} 63-\$ \mathrm{H} \$ 59)$

J63: (F2) (H64 - H63)

K63: (F2) [W12] (H63 - H61)

L63: (F2) (D63*\$D $\$ 8)^{*} 60$

M63: (F2) [W11] ( $\$ F \$ 52-\mathrm{H} 63) /(\$ F \$ 52$ - $\$ \mathrm{G} \$ 52)$

D64: (F4) $[\mathrm{W} 12]+\mathrm{D} 63+69.44$

E64: (F4) [W10] 1.0552

F64: (F4) $[\mathrm{W} 12]+\$ D \$ 8^{*} \mathrm{D} 64$

G64: (F2) [W11] +\$E $\$ 8^{*} \mathrm{E} 64$

H64: (F2) [W12] +\$E $\$ 8^{*} \mathrm{E} 64^{*} 14.223$

I64: (F2) [W12] @ ABS(H64 - \$H\$59)

J64: (F2) (H65 - H64)

K64: (F2) [W12] (H64 - H62)

L64: (F2) $\left(\mathrm{D} 64^{*} \$ \mathrm{D} \$ 8\right)^{*} 60$

M64: (F2) [W11] (\$F $\$ 52$ - H64)/( $\$ F \$ 52$ - \$G\$52)

D65: (F4) $[\mathrm{W} 12]+\mathrm{D} 64+69.44$

E65: (F4) [W10] 1.07318
F65: (F4) $[\mathrm{W} 12]+\$ \mathrm{D} \$ 8^{*} \mathrm{D} 65$

G65: (F2) $[\mathrm{W} 11]+\$ \mathrm{E} \$ 8^{*} \mathrm{E} 65$

H65: (F2) $[\mathrm{W} 12]+\$ \mathrm{E} \$ 8^{*} \mathrm{E} 65^{*} 14.223$

I65: (F2) [W12] @ ABS $(\mathrm{H} 65-\$ \mathrm{H} \$ 59)$

J65: (F2) (H66 - H65)

K65: (F2) [W12] (H65 - H63)

L65: (F2) $\left(\mathrm{D} 65^{*} \$ \mathrm{D} \$ 8\right)^{*} 60$

M65: (F2) [W11] ( $\$ F \$ 52$ - H65) / $\$ F \$ 52$ - \$G\$52)

D66: (F4) $[\mathrm{W} 12]+\mathrm{D} 65+69.44$

E66: (F4) [W10] 1.0908

F66: (F4) $[\mathrm{W} 12]+\$ D \$ 8^{*} \mathrm{D} 66$

G66: (F2) [W11] $+\$ E \$ 8^{*} \mathrm{E} 66$

$\mathrm{H} 66:(\mathrm{F} 2)[\mathrm{W} 12]+\$ \mathrm{E} \$ 8^{*} \mathrm{E} 66^{*} 14.223$

I66: (F2) [W12] @ ABS(H66 - \$H\$59)

J66: (F2) (H67 - H66)

K66: (F2) [W12] (H66 - H64)

L66: (F2) $\left(\mathrm{D} 66^{*} \$ \mathrm{D} \$ 8\right)^{*} 60$

M66: (F2) [W11] (\$F\$52 - H66)/(\$F\$52 - \$G\$52)

D67: (F4) $[\mathrm{W} 12]+\mathrm{D} 66+69.44$

E67: (F4) [W10] 1.1072

F67: (F4) [W12] +\$D\$8* D67

G67: (F2) $[\mathrm{W} 11]+\$ \mathrm{E} \$ 8^{*} \mathrm{E} 67$

$\mathrm{H} 67:(\mathrm{F} 2)[\mathrm{W} 12]+\$ \mathrm{E} \$ 8^{*} \mathrm{E} 67^{*} 14.223$

I67: (F2) [W12] @ ABS $(\mathrm{H} 67-\$ \mathrm{H} \$ 59)$

J67: (F2) (H68 - H67)

K67: (F2) [W12] (H67 - H65)

L67: (F2) $\left(\mathrm{D} 67^{*} \$ \mathrm{D} \$ 8\right)^{*} 60$

M67: (F2) [W11] (\$F $\$ 52$ - H67)/(\$F $\$ 52$ - \$G\$52)

D68: (F4) $[\mathrm{W} 12]+\mathrm{D} 67+69.44$

E68: (F4) [W10] 1.12196

F68: (F4) $[\mathrm{W} 12]+\$ D \$ 8^{*} \mathrm{D} 68$

G68: (F2) $[\mathrm{W} 11]+\$ \mathrm{E} \$ 8^{*} \mathrm{E} 68$

$\mathrm{H} 68:(\mathrm{F} 2)[\mathrm{W} 12]+\$ \mathrm{E} \$ 8^{*} \mathrm{E} 68^{*} 14.223$

I68: (F2) [W12] @ ABS $(\mathrm{H} 68-\$ \mathrm{H} \$ 59)$

J68: (F2) (H69 - H68)

K68: (F2) [W12] (H68 - H66)

L68: (F2) $\left(\mathrm{D} 68^{*} \$ \mathrm{D} \$ 8\right)^{*} 60$

M68: (F2) [W11] (\$F $\$ 52-\mathrm{H} 68) /(\$ \mathrm{~F} \$ 52$ - $\$ \mathrm{G} \$ 52)$

D69: (F4) $[\mathrm{W} 12]+\mathrm{D} 68+69.44$

E69: (F4) [W10] 1.1363

F69: (F4) $[\mathrm{W} 12]+\$ \mathrm{D} \$ 8^{*} \mathrm{D} 69$

G69: (F2) $[\mathrm{W} 11]+\$ E \$ 8^{*} \mathrm{E} 69$

$\mathrm{H} 69:(\mathrm{F} 2)[\mathrm{W} 12]+\$ \mathrm{E} \$ 8^{*} \mathrm{E} 69^{*} 14.223$

I69: (F2) [W12] @ $\mathrm{ABS}(\mathrm{H} 69-\$ \mathrm{H} \$ 59)$

J69: (F2) (H70 - H69)

K69: (F2) [W12] (H69 - H67)

L69: (F2) $\left(\mathrm{D} 69^{*} \$ \mathrm{D} \$ 8\right)^{*} 60$

M69: (F2) [W11] (\$F $\$ 52$ - H69)/ $\$$ F $\$ 52$ - \$G\$52)

D70: (F4) $[\mathrm{W} 12]+\mathrm{D} 69+69.44$

E70: (F4) [W10] 1.1501

F70: (F4) $[\mathrm{W} 12]+\$ \mathrm{D} \$ 8^{*} \mathrm{D} 70$

G70: (F2) $[\mathrm{W} 11]+\$ \mathrm{E} \$ 8^{*} \mathrm{E} 70$

$\mathrm{H} 70:(\mathrm{F} 2)[\mathrm{W} 12]+\$ \mathrm{E} \$ 8^{*} \mathrm{E} 70^{*} 14.223$

I70: (F2) [W12] @ $\mathrm{ABS}(\mathrm{H} 70$ - \$H\$59)

J70: (F2) (H71 - H70)

K70: (F2) [W12] (H70 - H68)

L70: (F2) $\left(\mathrm{D} 70^{*} \$ \mathrm{D} \$ 8\right)^{*} 60$

M70: (F2) [W11] (\$F\$52 - H70)/(\$F\$52 - \$G\$52)

D71: (F4) $[\mathrm{W} 12]+\mathrm{D} 70+69.44$

E71: (F4) [W10] 1.1632 
F71: (F4) [W12] +\$D\$8*D71

G71: (F2) [W11] $+\$ \mathrm{E} \$ 8^{*} \mathrm{E} 71$

H71: (F2) [W12] + $\$ \mathrm{E} \$ 8^{*} \mathrm{E} 71^{*} 14.223$

I71: (F2) [W12] @ ABS $(\mathrm{H} 71-\$ \mathrm{H} \$ 59)$

J71: (F2) (H72 - H71)

K71: (F2) [W12] (H71 - H69)

L71: (F2) (D71*\$D $\$ 8)^{*} 60$

M71: (F2) [W11] (\$F \$52 - H71)/( $\$ \mathrm{~F} \$ 52$ - \$G\$52)

D72: (F4) $[\mathrm{W} 12]+\mathrm{D} 71+69.44$

E72: (F4) [W10] 1.176

F72: (F4) $[\mathrm{W} 12]+\$ \mathrm{D} \$ 8^{*} \mathrm{D} 72$

G72: (F2) $[\mathrm{W} 11]+\$ \mathrm{E} \$ 8^{*} \mathrm{E} 72$

H72: (F2) $[\mathrm{W} 12]+\$ \mathrm{E} \$ 8^{*} \mathrm{E} 72^{*} 14.223$

I72: (F2) [W12] @ ABS(H72 - \$H\$59)

J72: (F2) (H73 - H72)

K72: (F2) [W12] (H72 - H70)

L72: (F2) $\left(\mathrm{D} 72^{*} \$ \mathrm{D} \$ 8\right)^{*} 60$

M72: (F2) [W11] (\$F\$52 - H72)/(\$F\$52 - \$G\$52)

D73: (F4) $[\mathrm{W} 12]+\mathrm{D} 72+69.44$

E73: (F4) [W10] 1.1879

F73: (F4) [W12] $+\$ D \$ 8^{*} \mathrm{D} 73$

G73: (F2) $[\mathrm{W} 11]+\$ \mathrm{E} \$ 8^{*} \mathrm{E} 73$

$\mathrm{H} 73:(\mathrm{F} 2)[\mathrm{W} 12]+\$ \mathrm{E} \$ 8^{*} \mathrm{E} 73^{*} 14.223$

I73: (F2) [W12] @ ABS(H73 - \$H\$59)

J73: (F2) (H74 - H73)

K73: (F2) [W12] (H73 - H71)

L73: (F2). (D73*\$D $\$ 8)^{*} 60$

M73: (F2) [W11] (\$F\$52 - H73)/( \$F\$52 - \$G\$52)

D74: (F4) $[\mathrm{W} 12]+\mathrm{D} 73+69.44$

E74: (F4) [W10] 1.1993

F74: (F4) $[\mathrm{W} 12]+\$ \mathrm{D} \$ 8^{*} \mathrm{D} 74$

G74: (F2) $[\mathrm{W} 11]+\$ E \$ 8^{*} \mathrm{E} 74$

H74: (F2) [W12] + $\$ \mathrm{E} \$ 8^{*} \mathrm{E} 74^{*} 14.223$

I74: (F2) [W12] @ ABS(H74 - \$H \$59)

J74: (F2) (H75 - H74)

K74: (F2) [W12] (H74 - H72)

L74: (F2) $\left(\mathrm{D} 74^{*} \$ \mathrm{D} \$ 8\right)^{*} 60$

M74: (F2) [W11] ( $\$ F \$ 52$ - H74)/( $\$ F \$ 52$ - \$G\$52)

D75: (F4) [W12] +D74+69.44

E75: (F4) [W10] 1.2105

F75: (F4) $[\mathrm{W} 12]+\$ \mathrm{D} \$ 8^{*} \mathrm{D} 75$

G75: (F2) $[\mathrm{W} 11]+\$ \mathrm{E} \$ 8^{*} \mathrm{E} 75$

H75: (F2) $[\mathrm{W} 12]+\$ \mathrm{E} \$ 8^{*} \mathrm{E} 75^{*} 14.223$

I75: (F2) [W12] @ ABS(H75 - \$H \$59)

J75: (F2) (H76 - H75)

K75: (F2) [W12] (H75 - H73)

L75: (F2) (D75*\$D $\$ 8)^{*} 60$

M75: (F2) [W11] (\$F\$52 - H75)/(\$F\$52 - \$G\$52)

D76: (F4) $[\mathrm{W} 12]+\mathrm{D} 75+69.44$

E76: (F4) [W10] 1.221

F76: (F4) $[\mathrm{W} 12]+\$ \mathrm{D} \$ 8^{*} \mathrm{D} 76$

G76: (F2) $[\mathrm{W} 11]+\$ E \$ 8^{*} \mathrm{E} 76$

H76: (F2) $[\mathrm{W} 12]+\$ \mathrm{E} \$ 8^{*} \mathrm{E} 76^{*} 14.223$

I76: (F2) [W12] @ ABS $(\mathrm{H} 76-\$ \mathrm{H} \$ 59)$

J76: (F2) (H77 - H76)

K76: (F2) [W12] (H76 - H74)

L76: (F2) $\left(\mathrm{D} 76^{*} \$ \mathrm{D} \$ 8\right)^{*} 60$

M76: (F2) [W11] (\$F\$52 - H76)/( \$F $\$ 52$ - \$G\$52)

D77: (F4) $[\mathrm{W} 12]+\mathrm{D} 76+69.44$

E77: (F4) [W10] 1.2313
F77: (F4) $[\mathrm{W} 12]+\$ \mathrm{D} \$ 8^{*} \mathrm{D} 77$

G77: (F2) $[\mathrm{W} 11]+\$ \mathrm{E} \$ 8^{*} \mathrm{E} 77$

H77: (F2) $[\mathrm{W} 12]+\$ \mathrm{E} \$ 8^{*} \mathrm{E} 77^{*} 14.223$

I77: (F2) [W12] @ ABS(H77 - \$H\$59)

J77: (F2) (H78 - H77)

K77: (F2) [W12] (H77 - H75)

L77: (F2) (D77* $\$ \mathrm{D} \$ 8)^{*} 60$

M77: (F2) [W11] (\$F\$52 - H77)/( \$F\$52 - \$G\$52)

D78: (F4) $[\mathrm{W} 12]+\mathrm{D} 77+69.44$

E78: (F4) [W10] 1.2412

F78: (F4) $[\mathrm{W} 12]+\$ \mathrm{D} \$ 8^{*} \mathrm{D} 78$

G78: (F2) $[\mathrm{W} 11]+\$ E \$ 8^{*} \mathrm{E} 78$

H78: (F2) [W12] $+\$ E \$ 8^{*} \mathrm{E} 78^{*} 14.223$

I78: (F2) [W12] @ ABS $(\mathrm{H} 78-\$ \mathrm{H} \$ 59)$

J78: (F2) (H79 - H78)

K78: (F2) [W12] (H78 - H76)

L78: (F2) $\left(\mathrm{D} 78^{*} \$ \mathrm{D} \$ 8\right)^{*} 60$

M78: (F2) [W11] (\$F\$52 - H78)/( \$F\$52 - \$G\$52)

D79: (F4) $[\mathrm{W} 12]+\mathrm{D} 78+69.44$

E79: (F4) [W10] 1.2502

F79: (F4) [W12] + $\$ \mathrm{D} \$ 8^{*} \mathrm{D} 79$

G79: (F2) $[\mathrm{W} 11]+\$ \mathrm{E} \$ 8^{*} \mathrm{E} 79$

H79: (F2) $[\mathrm{W} 12]+\$ E \$ 8^{*} \mathrm{E} 79^{*} 14.223$

I79: (F2) [W12] @ $\mathrm{ABS}(\mathrm{H} 79-\$ \mathrm{H} \$ 59)$

J79: (F2) (H80 - H79)

K79: (F2) [W12] (H79 - H77)

L79: (F2) $\left(\mathrm{D} 79^{*} \$ \mathrm{D} \$ 8\right)^{*} 60$

M79: (F2) [W11] (\$F\$52 - H79)/(\$F\$52 - \$G\$52)

D80: (F4) [W12] +D79+69.44

E80: (F4) [W10] 1.2592

F80: (F4) $[\mathrm{W} 12]+\$ D \$ 8^{*} \mathrm{D} 80$

G80: (F2) $[\mathrm{W} 11]+\$ \mathrm{E} \$ 8^{*} \mathrm{E} 80$

$\mathrm{H} 80:(\mathrm{F} 2)[\mathrm{W} 12]+\$ \mathrm{E} \$ 8^{*} \mathrm{E} 80^{*} 14.223$

I80: (F2) [W12] @ $\mathrm{ABS}(\mathrm{H} 80-\$ \mathrm{H} \$ 59)$

J80: (F2) (H81 - H80)

K80: (F2) [W12] (H80 - H78)

L80: (F2) $\left(\mathrm{D} 80^{*} \$ \mathrm{D} \$ 8\right)^{*} 60$

M80: (F2) [W11] (\$F\$52 - H80)/(\$F\$52 - \$G\$52)

D81: (F4) [W12] +D80+69.44

E81: (F4) [W10] 1.2675

F81: (F4) $[\mathrm{W} 12]+\$ D \$ 8^{*} \mathrm{D} 81$

G81: (F2) $[\mathrm{W} 11]+\$ \mathrm{E} \$ 8^{*} \mathrm{E} 81$

H81: (F2) $[\mathrm{W} 12]+\$ \mathrm{E} \$ 8^{*} \mathrm{E} 81^{*} 14.223$

I81: (F2) [W12] @ ABS(H81 - \$H\$59)

J81: (F2) (H82 - H81)

K81: (F2) [W12] (H81 - H79)

L81: (F2) (D81* $\$ \mathrm{D} \$ 8)^{*} 60$

M81: (F2) [W11] (\$F\$52 - H81)/( $\$ F \$ 52$ - \$G\$52)

D82: (F4) $[\mathrm{W} 12]+\mathrm{D} 81+69.44$

E82: (F4) [W10] 1.2758

F82: (F4) $[\mathrm{W} 12]+\$ \mathrm{D} \$ 8^{*} \mathrm{D} 82$

G82: (F2) $[\mathrm{W} 11]+\$ E \$ 8^{*} \mathrm{E} 82$

H82: (F2) [W12] $+\$ E \$ 8 * \mathrm{E} 82^{*} 14.223$

I82: (F2) [W12] @ ABS(H82 - \$H\$59)

J82: (F2) (H83 - H82)

K82: (F2) [W12] (H82 - H80)

L82: (F2) $\left(\mathrm{D} 82^{*} \$ \mathrm{D} \$ 8\right)^{*} 60$

M82: (F2) [W11] ( $\$ F \$ 52$ - H82)/( $\$ F \$ 52$ - \$G\$52)

D83: (F4) $[\mathrm{W} 12]+\mathrm{D} 82+69.44$

E83: (F4) [W10] 1.2836 
F83: (F4) $[\mathrm{W} 12]+\$ \mathrm{D} \$ 8^{*} \mathrm{D} 83$

G83: (F2) $[\mathrm{W} 11]+\$ \mathrm{E} \$ 8^{*} \mathrm{E} 83$

H83: (F2) [W12] $+\$ \mathrm{E} \$ 8^{*} \mathrm{E} 83^{*} 14.223$

I83: (F2) [W12] @ABS(H83 - \$H\$59)

J83: (F2) (ERR - H83)

K83: (F2) [W12] (H83 - H81)

L83: (F2) $\left(\mathrm{D} 83^{*} \$ \mathrm{D} \$ 8\right)^{*} 60$

M83: (F2) [W11] ( $\$ F \$ 52-\mathrm{H} 83) /(\$ F \$ 52$ - $\$ \mathrm{G} \$ 52)$

A87: [W23] 'BEAN STUDY: OIL WELL

B90: [W14]' B

C90: [W12]' C

D90: (F2) [W12] ^D

E90: (F2) [W10] ${ }^{\wedge} \mathrm{E}$

F90: (F2) [W12] ${ }^{\wedge} \mathrm{F}$

G90: (F2) [W11] ^ $\mathrm{G}$

H90: [W12] ${ }^{\wedge} \mathrm{H}$

I90: $[\mathrm{W} 12]^{\wedge} \mathrm{I}$

J90: ' J

B91: [W14]' DATE

C91: [W12] 'BEAN SIZE

D91: (F2) [W12] `DEL. TIME

E91: (F2) [W10] ^ PRESSURE

F91: (F2) [W12] ^DEL. TIME

G91: (F2) [W11] ^ PRESSURE

H91: [W12] ^ PRESSURE

I91: [W12] ^ DEL - P

J91: 'RATE

D92: (F2) [W12] ^div.

E92: (F2) [W10] $\mathrm{mm}$

F92: (F2) [W12] ${ }^{\wedge} \mathrm{hr}$

G92: (F2) [W11] ${ }^{\wedge} \mathrm{kg} / \mathrm{cm} 2$

H92: [W12] ^ psi

I92: [W12] ${ }^{\wedge} \mathrm{psi}$

J92:' $\mathrm{m} 3 / \mathrm{d}$

B94: [W14] '07.08.1985

C94: [W12] 0

D94: [W12] 4219

E94: [W10] 1.184

F94: (F4) $[\mathrm{W} 12]+\$ \mathrm{D} \$ 8^{*} \mathrm{D} 94$

G94: (F2) $[\mathrm{W} 11]+\$ E \$ 8^{*} \mathrm{E} 94$

H94: (F2) [W12] +\$E $\$ 8^{*} \mathrm{E} 94^{*} 14.223$

I94: (F2) [W12] + H94 - \$H $\$ 94$

J94: 0

B95: [W14] '08.08.1985

C95: [W12] 3

D95: [W12] 1100

E95: (F4) [W10] 1.14

F95: (F4) $[\mathrm{W} 12]+\$ D \$ 8^{*} \mathrm{D} 95$

G95: (F2) $[\mathrm{W} 11]+\$ E \$ 8^{*} \mathrm{E} 95$

H95: (F2) $[\mathrm{W} 12]+\$ \mathrm{E} \$ 8^{*} \mathrm{E} 95^{*} 14.223$

I95: (F2) [W12] + H95 - \$H $\$ 94$

J95: (F2) 20

B96: [W14] '09.08.1985

C96: [W12] 4

D96: [W12] 1300

E96: (F4) [W10] 1.096

F96: (F4) [W12] +\$D\$8*D96

G96: (F2) $[\mathrm{W} 11]+\$ \mathrm{E} \$ 8^{*} \mathrm{E} 96$

H96: (F2) [W12] +\$E $\$ 8^{*} \mathrm{E} 96^{*} 14.223$

I96: (F2) $[\mathrm{W} 12]+\mathrm{H} 96-\$ \mathrm{H} \$ 94$
J96: (F2) 30

B97: (D1) [W14] '10.08.1985

C97: [W12] 5

D97: [W12] 1500

E97: (F4) [W10] 1.052

F97: (F4) $[\mathrm{W} 12]+\$ \mathrm{D} \$ 8^{*} \mathrm{D} 97$

G97: (F2) [W11] + $\$ \mathrm{E} \$ 8^{*} \mathrm{E} 97$

H97: (F2) [W12] +\$E $\$ 8^{*} \mathrm{E} 97^{*} 14.223$

I97: (F2) [W12] +H97 - \$H $\$ 94$

J97: (F2) 40

B98: (D1) [W14] '11.08.1985

C98: [W12] 6

D98: [W12] 1700

E98: (F4) [W10] 0.947

F98: (F4) [W12] $+\$ D \$ 8^{*} \mathrm{D} 98$

G98: (F2) [W11] +\$E $\$ 8^{*} \mathrm{E} 98$

H98: (F2) $[\mathrm{W} 12]+\$ \mathrm{E} \$ 8^{*} \mathrm{E} 98^{*} 14.223$

I98: (F2) [W12] + H98 - $\$ \mathrm{H} \$ 94$

J98: (F2) 50

B99: (D1) [W14] '12.08.1985

C99: [W12] 7

D99: [W12] 1800

E99: (F4) [W10] 0.833

F99: (F4) $[\mathrm{W} 12]+\$ \mathrm{D} \$ 8^{*} \mathrm{D} 99$

G99: (F2) [W11] + \$E $\$ 8^{*} \mathrm{E} 99$

H99: (F2) [W12] +\$E $\$ 8^{*} \mathrm{E} 99^{*} 14.223$

I99: (F2) [W12] + H99 - $\$ \mathrm{H} \$ 94$

J99: (F2) 60

A103: [W23] 'BEAN STUDY: GAS WELL

F103: [W12] 'Ps2 - 1.033=

G103: [W11] +G110*G110 - 1.033

H103: [W12] ' $\mathrm{kg} / \mathrm{cm} 2$

B106: [W14]' B

C106: [W12]' C

D106: (F2) [W12] ^D

E106: (F2) [W10] ^ $\mathrm{E}$

F106: (F2) [W12] $\wedge \mathrm{F}$

G106: (F2) [W11] ${ }^{\wedge} \mathrm{G}$

H106: [W12] ^ $\mathrm{H}$

I106: [W12] ^I

J106: ' J

B107: [W14]' DATE

C107: [W12] 'BEAN SIZE

D107: (F2) [W12] ^DEL. TIME

E107: (F2) [W10] ^PRESSURE

F107: (F2) [W12] ^ DEL. TIME

G107: (F2) [W11] ^ PRESSURE

H107: [W12] ^ PRESSURE

I107: [W12] 'PS2 - PF2

J107: 'RATE

D108: (F2) [W12] ^div.

E108: (F2) [W10] $\wedge \mathrm{mm}$

F108: (F2) [W12] ${ }^{\wedge} \mathrm{hr}$

G108: (F2) [W11] ${ }^{\wedge} \mathrm{kg} / \mathrm{cm} 2$

H108: [W12] ^ psi

I108: [W12] ' $\mathrm{kg} / \mathrm{cm} 2$

J108: ' m3/d

B110: [W14] '07.08.1985

C110: [W12] 0

D110: [W12] 4219 
E110: [W10] 1.8063

F110: (F4) [W12] + $\$ \mathrm{D} \$ 8^{*} \mathrm{D} 110$

G110: (F2) [W11] +\$E $\$ 8^{*} \mathrm{E} 110$

H110: (F2) [W12] + $\$ E \$ 8^{*} \mathrm{E} 110^{*} 14.223$

B111: [W14] '08.08.1985

C111: [W12] 3

D111: [W12] 1100

E111: [W10] 1.6655

F111: (F4) [W12] +\$D $\$ 8^{*} \mathrm{D} 111$

G111: (F2) [W11] + $\$ \mathrm{E} \$ 8^{*} \mathrm{E} 111$

H111: (F2) $[\mathrm{W} 12]+\$ \mathrm{E} \$ 8^{*} \mathrm{E} 111^{*} 14.223$

I111: (F2) [W12] (\$G\$110*\$G\$110) - (G111*G111)

J111: 18000

B112: [W14] '09.08.1985

C112: [W12] 4

D112: [W12] 1300

E112: [W10] 1.6399

F112: (F4) [W12] + \$D $\$ 8^{*} \mathrm{D} 112$

G112: (F2) [W11] +\$E\$8*E112

H112: (F2) $[\mathrm{W} 12]+\$ \mathrm{E} \$ 8^{*} \mathrm{E} 112^{*} 14.223$

I112: (F2) [W12] ( $\$$ G $\left.\$ 110^{*} \$ \mathrm{G} \$ 110\right)$ - (G112*G112)

J112: 23000

B113: (D1) [W14] '10.08.1985

C113: [W12] 5

D113: [W12] 1500

E113: [W10] 1.4962

F113: (F4) [W12] + \$D $\$ 8^{*} \mathrm{D} 113$

G113: (F2) $[\mathrm{W} 11]+\$ \mathrm{E} \$ 8^{*} \mathrm{E} 113$

H113: (F2) $[\mathrm{W} 12]+\$ E \$ 8^{*} \mathrm{E} 113^{*} 14.223$

I113: (F2) [W12] (\$G\$110*\$G\$110) - (G113*G113)

J113: 36000

B114: (D1) [W14] '11.08.1985

C114: [W12] 6

D114: [W12] 1700

E114: [W10] 1.3469

F114: (F4) [W12] +\$D $\$ 8^{*} \mathrm{D} 114$

G114: (F2) $[\mathrm{W} 11]+\$ \mathrm{E} \$ 8^{*} \mathrm{E} 114$

H114: (F2) $[\mathrm{W} 12]+\$ \mathrm{E} \$ 8^{*} \mathrm{E} 114^{*} 14.223$

I114: (F2) [W12] (\$G\$110*\$G $\$ 110)-\left(\mathrm{G} 114^{*} \mathrm{G} 114\right)$

J114: 47000

B115: (D1) [W14] '12.08.1985

C115: [W12] 7

D115: [W12] 1800

E115: [W10] 1.1719

F115: (F4) [W12] + \$D $\$ 8^{*} \mathrm{D} 115$

G115: (F2) [W11] + $\$ \mathrm{E} \$ 8^{*} \mathrm{E} 115$

H115: (F2) [W12] + $\$ \mathrm{E} \$ 8^{*} \mathrm{E} 115^{*} 14.223$

I115: (F2) [W12] (\$G\$110*\$G\$110) - (G115*G115)

J115: 60000

A119: [W23] 'MODIFIED ISO-CHRONAL TEST: GAS WELL

C120: [W12] 'Date: 8.8 .88

F120: [W12] 'Ps2 - 1.033=

G120: (F2) [W11] + $\$$ G $\$ 127^{*} \$ \mathrm{G} \$ 127-1.033$

H120: [W12] 'kg/cm2

C123: [W12]' C

D123: (F2) [W12] ^ D

E123: (F2) [W10] ${ }^{\wedge} \mathrm{E}$

F123: (F2) [W12] ^ $\mathrm{F}$

G123: (F2) [W11] ${ }^{\wedge} \mathrm{G}$
H123: [W12] ^ $\mathrm{H}$

I123: [W12] ^ I

J123: ' J

C124: [W12] 'BEAN SIZE

D124: (F2) [W12] ` DEL. TIME

E124: (F2) [W10] ^ PRESSURE

F124: (F2) [W12] ^ DEL. TIME

G124: (F2) [W11] ^ PRESSURE

H124: [W12] ^PRESSURE

I124: [W12] 'PS2 - PF2

J124: 'RATE

D125: (F2) [W12] ^ div.

E125: (F2) [W10] ^ $\mathrm{mm}$

F125: (F2) [W12] ^ $\mathrm{hr}$

G125: (F2) [W11] ^ $\mathrm{kg} / \mathrm{cm} 2$

H125: [W12] ^ $\mathrm{psi}$

I125: [W12] ' kg/cm2

J125:' $\mathrm{m} 3 / \mathrm{d}$

C127: [W12]' 3(Pws)

D127: [W12] 0

E127: [W10] 2.69

F127: (F2) [W12] + \$D $\$ 8^{*} \mathrm{D} 127$

G127: (F2) [W11] + $\$ \mathrm{E} \$ 8^{*} \mathrm{E} 127$

H127: (F2) [W12] + $\$ E \$ 8^{*} \mathrm{E} 127^{*} 14.223$

D128: [W12] 69.44

E128: [W10] 2.545

F128: (F2) [W12] + \$D $\$ 8^{*} \mathrm{D} 128$

G128: (F2) [W11] + $\$ \mathrm{E} \$ 8^{*} \mathrm{E} 128$

H128: (F2) [W12] + $\$ \mathrm{E} \$ 8^{*} \mathrm{E} 128^{*} 14.223$

I128: (F2) [W12] ( $\$$ G $\left.\$ 127^{*} \$ \mathrm{G} \$ 127\right)-\left(\mathrm{G} 128^{*} \mathrm{G} 128\right)$

J128: 30000

D129: [W12] 138.88

E129: [W10] 2.4828

F129: (F2) [W12] + $\$ \mathrm{D} \$ 8^{*} \mathrm{D} 129$

G129: (F2) [W11] + $\$ \mathrm{E} \$ 8^{*} \mathrm{E} 129$

H129: (F2) [W12] + $\$ \mathrm{E} \$ 8^{*} \mathrm{E} 129^{*} 14.223$

I129: (F2) [W12] ( $\$$ G $\left.\$ 127^{*} \$ \mathrm{G} \$ 127\right)-\left(\mathrm{G} 129^{*} \mathrm{G} 129\right)$

J129: 30000

D130: [W12] 208.32

E130: [W10] 2.4104

F130: (F2) [W12] $+\$ D \$ 8^{*} \mathrm{D} 130$

G130: (F2) [W11] +\$E $\$ 8^{*} \mathrm{E} 130$

$\mathrm{H} 130:(\mathrm{F} 2)[\mathrm{W} 12]+\$ \mathrm{E} \$ 8^{*} \mathrm{E} 130^{*} 14.223$

I130: (F2) [W12] ( $\$$ G $\left.\$ 127^{*} \$ \mathrm{G} \$ 127\right)$ - $\left(\mathrm{G} 130^{*} \mathrm{G} 130\right)$

J130: 30000

D131: [W12] 277.76

E131: [W10] 2.3331

F131: (F2) [W12] +\$D\$8*D131

G131: (F2) [W11] +\$E\$8*E131

$\mathrm{H} 131:(\mathrm{F} 2)[\mathrm{W} 12]+\$ \mathrm{E} \$ 8^{*} \mathrm{E} 131^{*} 14.223$

I131: (F2) [W12] ( $\$$ G $\left.\$ 127^{*} \$ \mathrm{G} \$ 127\right)-\left(\mathrm{G} 131^{*} \mathrm{G} 131\right)$

J131: 30000

C132: [W12]' 4(Pws*)

D132: [W12] 0

E132: [W10] 2.645

F132: (F2) [W12] +\$D $\$ 8^{*} \mathrm{D} 132$

G132: (F2) $[\mathrm{W} 11]+\$ \mathrm{E} \$ 8^{*} \mathrm{E} 132$

H132: (F2) $[\mathrm{W} 12]+\$ \mathrm{E} \$ 8^{*} \mathrm{E} 132^{*} 14.223$

D133: [W12] 69.44

E133: [W10] 2.3705 
F133: (F2) [W12] + $\$ \mathrm{D} \$ 8^{*} \mathrm{D} 133$

G133: (F2) [W11] $+\$ \mathrm{E} \$ 8^{*} \mathrm{E} 133$

H133: (F2) $[\mathrm{W} 12]+\$ \mathrm{E} \$ 8^{*} \mathrm{E} 133^{*} 14.223$

I133: (F2) [W12] (\$G\$132*\$G\$132) - (G133*G133)

J133: 46000

D134: [W12] 138.88

E134: [W10] 2.2333

F134: (F2) [W12] + $\$ \mathrm{D} \$ 8^{*} \mathrm{D} 134$

G134: (F2) [W11] + $\$ E \$ 8^{*} \mathrm{E} 134$

H134: (F2) [W12] + $\$ E \$ 8^{*} \mathrm{E} 134^{*} 14.223$

I134: (F2) [W12] (\$G\$132*\$G\$132) - (G134*G134)

J134: 46000

D135: [W12] 208.32

E135: [W10] 2.0836

F135: (F2) [W12] + $\$ D \$ 8^{*} \mathrm{D} 135$

G135: (F2) [W11] $+\$ E \$ 8^{*} \mathrm{E} 135$

H135: (F2) [W12] + $\$ \mathrm{E} \$ 8^{*} \mathrm{E} 135^{*} 14.223$

I135: (F2) [W12] ( $\$$ G $\left.\$ 132^{*} \$ \mathrm{G} \$ 132\right)-\left(\mathrm{G} 135^{*} \mathrm{G} 135\right)$

J135: 46000

D136: [W12] 277.76

E136: [W10] 1.9463

F136: (F2) [W12] + $\$ \mathrm{D} \$ 8^{*} \mathrm{D} 136$

G136: (F2) $[\mathrm{W} 11]+\$ \mathrm{E} \$ 8^{*} \mathrm{E} 136$

H136: (F2) [W12] + $\$ E \$ 8^{*} \mathrm{E} 136^{*} 14.223$

I136: (F2) [W12] ( $\$$ G $\left.\$ 132^{*} \$ \mathrm{G} \$ 132\right)-\left(\mathrm{G} 136^{*} \mathrm{G} 136\right)$

J136: 46000

C137: [W12]' 5(Pws*)

D137: [W12] 0

E137: [W10] 2.6076

F137: (F2) [W12] + \$D\$8*D137

G137: (F2) [W11] +\$E\$8*E137

H137: (F2) [W12] + $\$ \mathrm{E} \$ 8^{*} \mathrm{E} 137^{*} 14.223$

D138: [W12] 69.44

E138: [W10] 2.2707

F138: (F2) [W12] + $\$ \mathrm{D} \$ 8^{*} \mathrm{D} 138$

G138: (F2) [W11] + $\$ \mathrm{E} \$ 8^{*} \mathrm{E} 138$

H138: (F2) $[\mathrm{W} 12]+\$ E \$ 8^{*} \mathrm{E} 138^{*} 14.223$

I138: (F2) [W12] ( $\$$ G $\left.\$ 137^{*} \$ \mathrm{G} \$ 137\right)-\left(\mathrm{G} 138^{*} \mathrm{G} 138\right)$

J138: 54000

D139: [W12] 138.88

E139: [W10] 2.101

F139: (F2) [W12] + \$D \$8*D139

G139: (F2) [W11] + $\$ \mathrm{E} \$ 8^{*} \mathrm{E} 139$

H139: (F2) $[\mathrm{W} 12]+\$ \mathrm{E} \$ 8^{*} \mathrm{E} 139^{*} 14.223$

I139: (F2) [W12] (\$G\$137*\$G\$137) - $\left(\mathrm{G} 139^{*} \mathrm{G} 139\right)$

J139: 54000

D140: [W12] 208.32

E140: [W10] 1.9089

F140: (F2) [W12] + \$D $\$ 8^{*} \mathrm{D} 140$

G140: (F2) $[\mathrm{W} 11]+\$ \mathrm{E} \$ 8^{*} \mathrm{E} 140$

H140: (F2) $[\mathrm{W} 12]+\$ E \$ 8^{*} \mathrm{E} 140^{*} 14.223$

I140: (F2) [W12] ( $\$$ G $\left.\$ 137^{*} \$ \mathrm{G} \$ 137\right)-\left(\mathrm{G} 140^{*} \mathrm{G} 140\right)$

J140: 54000

D141: [W12] 277.76

E141: [W10] 1.7217

F141: (F2) [W12] $+\$ \mathrm{D} \$ 8^{*} \mathrm{D} 141$

G141: (F2) [W11] +\$E\$8*E141

H141: (F2) [W12] + $\$ \mathrm{E} \$ 8^{*} \mathrm{E} 141^{*} 14.223$

I141: (F2) [W12] ( $\$$ G $\left.\$ 137^{*} \$ \mathrm{G} \$ 137\right)$ - $\left(\mathrm{G} 141^{*} \mathrm{G} 141\right)$

J141: 54000
C142: [W12]' 6(Pws*)

D142: [W12] 0

E142: [W10] 2.5826

F142: (F2) [W12] + \$D $\$ 8^{*} \mathrm{D} 142$

G142: (F2) $[\mathrm{W} 11]+\$ \mathrm{E} \$ 8^{*} \mathrm{E} 142$

H142: (F2) [W12] +\$E $\$ 8^{*} \mathrm{E} 142^{*} 14.223$

D143: [W12] 69.44

E143: [W10] 2.1959

F143: (F2) [W12] +\$D $\$ 8^{*} \mathrm{D} 143$

G143: (F2) [W11] + $\$ \mathrm{E} \$ 8^{*} \mathrm{E} 143$

H143: (F2) [W12] $+\$ E \$ 8^{*} \mathrm{E} 143^{*} 14.223$

I143: (F2) [W12] (\$G\$142*\$G\$142) - (G143*G143)

J143: 62000

D144: [W12] 138.88

E144: [W10] 1.9962

F144: (F2) [W12] +\$D\$8*D144

G144: (F2) [W11] $+\$ \mathrm{E} \$ 8^{*} \mathrm{E} 144$

H144: (F2) [W12] $+\$ E \$ 8^{*} \mathrm{E} 144^{*} 14.223$

I144: (F2) [W12] (\$G\$142*\$G\$142) - (G144*G144)

J144: 62000

D145: [W12] 208.32

E145: [W10] 1.7966

F145: (F2) $[\mathrm{W} 12]+\$ D \$ 8^{*} \mathrm{D} 145$

G145: (F2) $[\mathrm{W} 11]+\$ \mathrm{E} \$ 8^{*} \mathrm{E} 145$

H145: (F2) [W12] +\$E\$8*E145*14.223

I145: (F2) [W12] (\$G\$142*\$G\$142) - (G145*G145)

J145: 62000

D146: [W12] 277.76

E146: [W10] 1.597

F146: (F2) [W12] +\$D\$8*D146

G146: (F2) [W11] + $\$ \mathrm{E} \$ 8^{*} \mathrm{E} 146$

H146: (F2) [W12] $+\$ E \$ 8^{*} \mathrm{E} 146^{*} 14.223$

I146: (F2) [W12] (\$G\$142*\$G\$142) - (G146*G146)

J146: 62000

C147: [W12]' Pwf

D147: [W12] 2771

E147: [W10] 1.497

F147: (F2) $[\mathrm{W} 12]+\$ D \$ 8^{*} \mathrm{D} 147$

G147: (F2) $[\mathrm{W} 11]+\$ \mathrm{E} \$ 8^{*} \mathrm{E} 147$

H147: (F2) [W12] $+\$ E \$ 8^{*} \mathrm{E} 147^{*} 14.223$

I147: (F2) [W12] (\$G\$127*\$G\$127) - (G147*G147)

J147: 62000

C150: [W12] '* - Pressure after fixed time

A153: [W23] 'BASIC INPUT DATA: FROM PVT \& PETROPHYSICAL STUDIES AND FROM GRAPHS

A156: [W23] ^viscosity (cp)

B156: [W14] "pay thick.h(m)

C156: (F2) [W12] "p1hr(psi)

I156: [W12] 'Steady time

K156: [W12] 'Beta(Muskat)

L156: 'Ia(ft3/day)for WRB

A157: (F2) [W23] 584

B157: (F2) [W14] 9

C157: (F2) [W12] 88.8*14.223

I157: [W12] 60.75

K157: (S4) [W12] 0.001111

L157: (S2) 1000000

A159: (F4) [W23] formation vol. factor

B159: (F0) [W14] ${ }^{\wedge} \mathrm{phi}(\%)$ 
C159: (F4) [W12] ^re(ft)for Muskat

I159: [W12]' Pwf(TRT)

K159: [W12] 'Beta(sssa)

L159: 'Ba(V/V) for WRB

A160: (F4) [W23] 1.0314

B160: (F2) [W14] 0.3

C160: (F2) [W12] 2154

I160: [W12] 82.4788*14.223

$\mathrm{K} 160$ : [W12] $\left(0.0418^{*} 26.54^{*} 0.001111\right) / 0.115$

L160: 0.0259

A162: (F4) [W23] ^slope pr.bu,dd,PFT( WI)

B162: (F4) [W14] $\mathrm{rw}(\mathrm{ft})$

C162: (F4) [W12] ^ p bar(psi)for Muskat

F162: [W12] ' $\mathrm{P}^{*}$ psi(PI cal.)

L162: 'Ca(1/psi) for WRB

A163: (F4) [W23] 14.233

B163: (F4) [W14] 0.354

C163: (F2) [W12] 1287.3

F163: (F1) [W12] 1285.96

L163: (S2) 0.00064

A165: (F4) [W23]^ b(Muskat)

B165: (F4) [W14] ${ }^{\wedge}$ ct (1/psi)

C165: (F4) [W12] ^ p^ (psi)for Muskat

F165: [W12] 'Pwf(psi)for W/I well

L165: 'porosity(\%)for WRB

A166: (F4) [W23] 26.5401

B166: (S4) [W14] 1.7297E - 06

C166: (F1) [W12] 1390

F166: [W12] 1286

L166: 0.3

A168: [W23] `slope(two rate test)

B168: [W14] ^ Pwf psi

C168: [W12] ^ Pst psi

F168: [W12] 'p bar(psi)for W/I well

L168: 'slope(psi/day)for WRB

A169: (F1) [W23] 14.233

B169: (F1) [W14] 1173.1

C169: (F1) [W12] 1285.96

F169: [W12] 1173.1

L169: 800

B172: [W14] 'RESULTS

A176: [W23]' A

B176: [W14], B

C176: [W12]' C

D176: (F2) [W12] ^ D

E176: (F2) [W10] ^ $\mathrm{E}$

F176: (F2) [W12] ^ $\mathrm{F}$

G176: (F2) [W11] ^ $\mathrm{G}$

H176: [W12] ^ $\mathrm{H}$

I176: [W12] ^ I

J176: (F2) ^ J

K176: [W12] ^ $\mathrm{K}$

L176: ^ L

M176: [W11] ^ M

N176: [W11] ` $\mathrm{N}$

O176: [W11] ^ $\mathrm{O}$

P176: [W11]'

A177: [W23], RESERVOIR - >

B177: $[\mathrm{W} 14]^{\wedge} \mathrm{Kh}$

C177: [W12] ^ $\mathrm{K}$
D177: [W12] ` Skin

E177: [W10] ^ Del-p

F177: [W12]' J actual

G177: [W11] $\mathrm{J}$ ideal

H177: (F2) [W12] ^ Flow eff.

I177: [W12] ^ Radius of

J177: 'P*(TRT)

K177: [W12] ` Pore vol

L177: 'BURNT VOL

M177: [W11] 'P static

N177: [W11] ^ $\mathrm{PI}$

O177: [W11] ^ OFP

P177: [W11]'

A178: [W23]' PARAMETERS

B178: [W14] ^ md.ft

C178: [W12] ^ $\mathrm{md}$

E178: [W10] ^ skin(psi)

F178: [W12] 'm3/d/ kg/cm2

G178: [W11] 'm3/d/ $\mathrm{kg} / \mathrm{cm} 2$

H178: (F2) [W12] ^\%

I178: [W12] ^ investi. m.

J178: ^ psi

K178: [W12] ` Res. bbl

L178: 'ft3

M178: [W11] ^ psi

N178: [W11] 'm3/d/kg/cm2

0178: [W11] ' $\mathrm{m} 3 / \mathrm{d}$

A180: [W23] 'MDH METHOD

B180: (F1) [W14] (162.6* $\left.\$ \mathrm{~K} \$ 8^{*} 6.289^{*} \$ \mathrm{~A} \$ 157^{*} \$ \mathrm{~A} \$ 160\right) /$ $(\$ A \$ 163)$

C180: (F1) [W12] (B180)/(\$B $\left.\$ 157^{*} 3.28\right)$

D180: (F1) [W12] 1.151* (( @ ABS $(\$ C \$ 157-\$ H \$ 16) /(\$$ $\mathrm{A} \$ 163))$ - @ LOG $\left(\$ C \$ 180 /\left(\$ B \$ 160^{*} \$ A \$ 157^{*} \$ B \$\right.\right.$ $\left.\left.\left.166^{*} \$ B \$ 163^{*} \$ B \$ 163\right)\right)+3.23\right)$ )

E180: (F1) [W10] 0.87*\$A $\$ 163^{*} \$ D \$ 180$

F180: (F1) [W12] (\$K\$8)/( $\$ F \$ 163 / 14.223)$ - (\$B $\$ 169 /$ 14.223))

G180: (F1) [W11] $(\$ K \$ 8) /((\$ F \$ 163 / 14.223)-(\$ B \$ 169 /$ $14.223)-(\$ \mathrm{E} \$ 180 / 14.223))$

H180: (F2) [W12] ( $\$$ F $180 \mathrm{G} \$ 180)$

I180: $(\mathrm{F} 1)[\mathrm{W} 12]\left(\left(\left(0.00105^{*} \$ \mathrm{C} \$ 180^{*} \$ \mathrm{I} \$ 157\right) /\left((\$ \mathrm{~B} \$ 160)^{*}\right.\right.\right.$ $\left.\left.\left.\left(\$ A \$ 157^{*} \$ B \$ 166\right)\right)\right)^{\wedge} 0.5\right) / 3.28$

A181: [W23] 'HORNER METHOD

B181: (F1) [W14] $\left(162.6^{*} \$ \mathrm{~K} \$ 8^{*} 6.289^{*} \$ \mathrm{~A} \$ 157^{*} \$ \mathrm{~A} \$ 160\right) /$ (\$A\$163)

C181: (F1) [W12] (B181)/(\$B $\left.\$ 157^{*} 3.28\right)$

D181: (F1) [W12] 1.151* (( @ ABS $(\$ C \$ 157-\$ H \$ 16) /(\$$ $\mathrm{A} \$ 163))$ - @ LOG $\left(\$ C \$ 181 /\left(\$ \mathrm{~B} \$ 160^{*} \$ \mathrm{~A} \$ 157^{*} \$ \mathrm{~B} \$\right.\right.$ $\left.\left.\left.\left.166^{*} \$ B \$ 163^{*} \$ B \$ 163\right)\right)+3.23\right)\right)$

E181: (F1) [W10] $0.87^{*} \$ A \$ 163^{*} \$ D \$ 181$

F181: (F1) [W12] (\$K\$8)/((\$F\$163/14.223) - (\$B \$169/ 14.223))

G181: (F1) [W11] ( $\$ \mathrm{~K} \$ 8) /((\$ \mathrm{~F} \$ 163 / 14.223)$ - ( $\$ \mathrm{~B} \$ 169 /$ $14.223)-(\$ \mathrm{E} \$ 181 / 14.223))$

H181: (F2) [W12] (\$F\$181G\$181)

I181: (F1) [W12] ( ( $\left.0.00105^{*} \$ \mathrm{C} \$ 181^{*} \$ \mathrm{I} \$ 157\right) /\left((\$ \mathrm{~B} \$ 160)^{*}\right.$ $\left.\left.\left.\left(\$ A \$ 157^{*} \$ \mathrm{~B} \$ 166\right)\right)\right)^{\wedge} 0.5\right) / 3.28$

A182: [W23] 'MUSKAT METHOD

B182: (F1) [W14] (118.6* $\left.\$ K \$ 8^{*} 6.289^{*} \$ A \$ 157^{*} \$ A \$ 160\right) /$ (\$A\$166) 
C182: (F1) [W12] (B182)/( $\left.\$ \mathrm{~B} \$ 157^{*} 3.28\right)$

D182: (F1) [W12] 0.84* $((\$ \mathrm{C} \$ 163-\$ \mathrm{C} \$ 166) /(\$ \mathrm{~A} \$ 166))$ $(2.3025)^{*} @ \operatorname{LOG}((\$ \mathrm{C} \$ 160) /(\$ \mathrm{~B} \$ 163))+3 / 4$

K182: (F0) [W12] 0.115* $\$ \mathrm{~K} \$ 8^{*} 6.289^{*} \$ \mathrm{~A} \$ 160 /\left(\$ \mathrm{~A} \$ 166^{*} \$\right.$ $\left.\mathrm{K} \$ 157^{*} \$ \mathrm{~B} \$ 166\right)$

A183: [W23] 'SEMI STEADY STATE ANA.

K183: (F0) [W12] 0.0418* $\$ \mathrm{~K} \$ 8^{*} 6.289^{*} \$ \mathrm{~A} \$ 160 /\left(\$ \mathrm{~K} \$ 160^{*}\right.$ $\$ \mathrm{~B} \$ 166)$

A184: [W23] 'PR. FALL OFF TEST(W/I)

B184: (F1) [W14] (162.6* $\left.\$ \mathrm{~K} \$ 8^{*} 6.289^{*} \$ \mathrm{~A} \$ 157^{*} \$ \mathrm{~A} \$ 160\right) /$ $(\$ A \$ 163)$

C184: (F1) [W12] (B184)/( $\left.\$ B \$ 157^{*} 3.28\right)$

D184: (F1) [W12] $1.151^{*}(((@ \mathrm{ABS}(\$ \mathrm{C} \$ 157-\$ \mathrm{H} \$ 16) /(\$$ $\mathrm{A} \$ 163))$ - @ LOG $\left(\$ C \$ 184 /\left(\$ \mathrm{~B} \$ 160^{*} \$ \mathrm{~A} \$ 157^{*} \$ \mathrm{~B} \$\right.\right.$ $\left.\left.\left.\left.166^{*} \$ \mathrm{~B} \$ 163^{*} \$ \mathrm{~B} \$ 163\right)\right)+3.23\right)\right)$

E184: (F1) [W10] 0.87*\$A $\$ 163^{*} \$ \mathrm{D} \$ 184$

F184: (F1) [W12] ( $\$ \mathrm{~K} \$ 8) /((\$ F \$ 166 / 14.223)-(\$ F \$ 169 /$ 14.223))

G184: (F1) [W11] $(\$ K \$ 8) /((\$ F \$ 166 / 14.223)-(\$ F \$ 169 /$ $14.223)-(\$ E \$ 184 / 14.223))$

H184: (F2) [W12] ( \$F \$184G\$184)

I184: (F1) [W12] $\left(\left(\left(0.025^{*} \$ C \$ 184^{*} \$ \mathrm{I} \$ 157\right) /\left((\$ B \$ 160)^{*}\right.\right.\right.$ $\left.\left.\left.\left(\$ A \$ 157^{*} \$ B \$ 166\right)\right)\right)^{\wedge} 0.5\right) / 3.28$

A185: [W23] 'TWO RATE TEST

B185: (F1) [W14] (162.6* $\left.\$ K \$ 8^{*} 6.289^{*} \$ \mathrm{~A} \$ 157^{*} \$ \mathrm{~A} \$ 160\right)$ $/(\$ A \$ 169)$

C185: (F1) [W12] (B185)/( $\left.\$ \mathrm{~B} \$ 157^{*} 3.28\right)$

D185: (F1) [W12] 1.151* ( ( $\$ \mathrm{~K} \$ 8) /(\$ \mathrm{~K} \$ 8-\$ \mathrm{~K} \$ 9)^{*}(\$ \mathrm{C} \$$ 157 - $\$$ B $\$ 169) /(\$ A \$ 169)$ - @ LOG $\left(\$ C \$ 185 /\left(\$ B \$ 160^{*}\right.\right.$ $\left.\left.\left.\left.\$ \mathrm{~A} \$ 157^{*} \$ \mathrm{~B} \$ 166^{*} \$ \mathrm{~B} \$ 163^{*} \$ \mathrm{~B} \$ 163\right)\right)+3.23\right)\right)$

E185: (F1) [W10] 0.87* $\$ A \$ 169^{*} \$ D \$ 185$

F185: (F1) [W12] ( $\$ \mathrm{~K} \$ 8) /((\$ F \$ 163 / 14.223)-(\$ B \$ 169 /$ 14.223))

G185: (F1) [W11] $(\$ K \$ 8) /((\$ F \$ 163 / 14.223)-(\$ B \$ 169 /$

14.223) - (\$E $\$ 185 / 14.223))$

H185: (F2) [W12] ( $\$ F \$ 185 G \$ 185)$

I185: (F1) [W12] '

J185: $(\mathrm{F} 1)+\$ \mathrm{I} \$ 160+(\$ A \$ 169)^{*}\left(@ \operatorname{LOG}\left(\left(\$ \mathrm{C} \$ 185^{*} \$ \mathrm{I} \$ 157\right)\right.\right.$ / $\left.\left(\$ \mathrm{~B} \$ 160^{*} \$ \mathrm{~A} \$ 157^{*} \$ \mathrm{~B} \$ 166^{*} \$ \mathrm{~B} \$ 163^{*} \$ \mathrm{~B} \$ 163\right)\right)-3.23$ $\left.+0.87^{*} \$ \mathrm{D} \$ 185\right)$

A186: [W23] 'WALSH RAMEY BRIGHAM MET.

L186: (S2) +\$L\$157*\$L $\$ 160 /\left(\$ \mathrm{~L} \$ 169^{*} \$ \mathrm{~L} \$ 166^{*} \$ \mathrm{~L} \$ 163\right)$

A187: [W23] 'INFLUX STUDY

M187: [W11] 1750

A188: [W23] 'BEAN STUDY (OIL WELL)

N188: [W11] 4.1

A189: [W23] 'BEAN STUDY (GAS WELL)

O189: [W11] 90000

A190: [W23] 'ISO - CHRONAL TEST

O190: [W11] 90000 